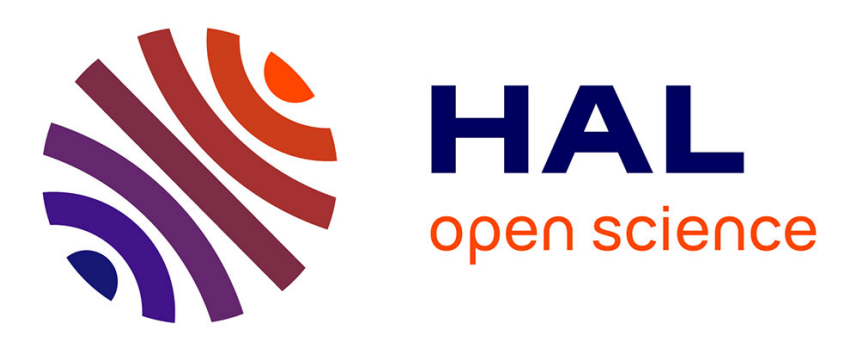

\title{
A critical assessment of flux and source term closures in shallow water models with porosity for urban flood simulations
}

\author{
Vincent Guinot
}

\section{- To cite this version:}

Vincent Guinot. A critical assessment of flux and source term closures in shallow water models with porosity for urban flood simulations. Advances in Water Resources, 2017, 109, pp.133-157. 10.1016/j.advwatres.2017.09.002 . hal-01582224

\section{HAL Id: hal-01582224 \\ https://hal.science/hal-01582224}

Submitted on 5 Sep 2017

HAL is a multi-disciplinary open access archive for the deposit and dissemination of scientific research documents, whether they are published or not. The documents may come from teaching and research institutions in France or abroad, or from public or private research centers.
L'archive ouverte pluridisciplinaire $\mathbf{H A L}$, est destinée au dépôt et à la diffusion de documents scientifiques de niveau recherche, publiés ou non, émanant des établissements d'enseignement et de recherche français ou étrangers, des laboratoires publics ou privés. 


\title{
A critical assessment of flux and source term closures in shallow water models with porosity for urban flood simulations
}

This is the preprint of an article accepted for publication in Advances in Water Resources. doi link: https://doi.org/10.1016/j.advwatres.2017.09.002

Vincent Guinot $(1,2)$

(1) Université de Montpellier, UMR HSM, CC57, 163 rue Auguste Broussonnet, 34090 Montpellier, France

(2) Inria Lemon, Bât 5 - CC05 017, 860 rue Saint-Priest, 34095 Montpellier Cedex 5, France

\begin{abstract}
The validity of flux and source term formulae used in shallow water models with porosity for urban flood simulations is assessed by solving the two-dimensional shallow water equations over computational domains representing periodic building layouts. The models under assessment are the Single Porosity (SP), the Integral Porosity (IP) and the Dual Integral Porosity (DIP) models. 9 different geometries are considered. 18 two-dimensional initial value problems and 6 two-dimensional boundary value problems are defined. This results in a set of 96 fine grid simulations. Analysing the simulation results leads to the following conclusions: (i) the DIP flux and source term models outperform those of the SP and IP models when the Riemann problem is aligned with the main street directions, (ii) all models give erroneous flux closures when is the Riemann problem is not aligned with one of the main street directions or when the main street directions are not orthogonal, (iii) the solution of the Riemann problem is self-similar in space-time when the street directions are orthogonal and the Riemann problem is aligned with one of them, (iv) a momentum balance confirms the existence of the transient momentum dissipation model presented in the DIP model, (v) none of the source term models presented so far in the literature allows all flow configurations to be accounted for(vi) future laboratory experiments aiming at the validation of flux and source term closures should focus on the high-resolution, two-dimensional monitoring of both water depth and flow velocity fields.
\end{abstract}

\section{Introduction}

Shallow water models with porosity have appeared over the past two decades as an efficient way of upscaling free surface flow problems in the presence of complex geometries. The main two application fields of such models are the simulation of shallow flows over natural surfaces (coastal lagoons and runoff over complex topography) and the fast simulation of urban floods. Four main types of twodimensional, porosity-based shallow water models are available from the literature.

The Single Porosity (SP) model was first proposed to account for subgrid scale topography [1, 5, 6, see e.g. [39] for a recent application to anisotropic topographical effects. Applications to urban flood modelling were proposed later [15, 16, 37. The porosity reflects the fraction of the plan view area available to mass and momentum storage. For shallow flows over natural topographies, the porosity is depth-dependent [1, 5, 6, 39, while urban flood applications consider a depth-independent porosity [15, 16, 37, 38. A number of numerical solution techniques have been developed for the depthindependent SP equations [2, 4, 8, 15, 26. Recently, a generalized source term has been proposed for the specific purposes of urban flood modelling [38].

The Integral Porosity (IP) model was introduced as a generalization of the SP model for the simulation of urban floods [34, 36]. Distinguishing between a storage porosity (isotropic) and a conveyance porosity (anisotropic) allows for a better description of the flow field than in the SP model [18, 19]. A depth-dependent version has appeared recently [29, 30]. The connectivity porosity, that is supposed 
to account for building obstruction to the flow, should be smaller than the storage porosity, otherwise yielding wave propagation speeds artificially larger than those in free water [13], an unphysical behaviour.

The Multiple Porosity (MP) model [1] was also developed to account for anisotropic effects. While the IP model used two different porosities over a given domain, the MP model uses a decomposition of the flow domain into several regions with different hydraulic properties: an immobile region, a mobile, isotropic region and several anisotropic regions where the flow is one-directional. The transfer of mass and momentum between these regions is governed by free surface elevation gradients. Specific momentum source term models are introduced to enforce the immobile and/or one-dimensional character of the flow. As a result, the wave propagation properties of the model do not obey an equation of state: for a given depth and flow velocity, assuming a rising or a falling water level yields different wave propagation speeds.

The Dual Integral Porosity (DIP) model [14] is a generalization of the IP model. In [14], the concept of closure is introduced. It stems from the consideration that the dual nature of the porosity (storage vs. porosity) should result in a dual definition of the flow variables (domain-based vs. boundary-based). The domain- and boundary-based flow variables must be related via a closure model. The IP model can be obtained as a particular case of the DIP formalism, by assuming a very specific closure. The IP closure yields inaccurate mass and momentum fluxes [14. Correcting this with the DIP closure and introducing a transient momentum dissipation model derived from [11 results in substantially improved model performance. A salient feature of the DIP model is that solution well-posedness requires that the connectivity porosity should be smaller than or equal to the storage porosity.

While the DIP model outperforms the SP and IP models when applied to one-dimensional problems, a two-dimensional application to a field scale test case (a $600 \mathrm{~m} \times 900 \mathrm{~m}$ neighbourhood near Sacramento) shows that both the IP and DIP model fail to reproduce the statistical distribution of the flow field exactly [14]. In particular, the fine grid simulation presented in [14] shows a strong polarization of the flow field along the main street axes. This is observed even though the buildings are not aligned exactly and their spacing is not regular. In contrast, the IP and DIP models are shown to produce more isotropic unit discharge and velocity fields under both transient and steady state. Moreover, a consistency and wave propagation analysis of the IP and DIP models [12] indicates that these models are sensitive to the design of the mesh in the presence of anisotropic connectivity porosity fields. The DIP model, however, is less sensitive than the IP model. Whether the problems identified in [14] stem from the consistency issues found in [12] is not clear at this stage.

The purpose of the present paper is to assess critically the flux closure and the source term models for the SP, IP and DIP models. The MP model cannot be assessed in the same way because it does not allow a unique flux function to be inferred from the domain-averaged variables. Therefore, it is excluded from the analysis. The performance of a model is usually assessed by comparing the simulation results to reference data, obtained from experimental sets or from simulations on refined grids. The model error, a measure of the difference between the simulation results and the reference data, may be appreciated using various indicators. Examples are the L-norms of the water depth, free surface elevations or velocity norm errors, or the Flood Extent Agreement (FEA) index [14, 36]. However, due to the competing influence of fluxes and source terms in the governing equations, model validation based on the sole simulation results is often indirect and incomplete. A model using erroneous fluxes and/or source terms may appear satisfactory provided that the calibration process allows an optimal balance between the various errors to be achieved. In the case of a poorly performing model, it is not possible to identify the reason for model failure (for instance, does model failure result from an erroneous flux model, or an erroneous source term model, or the combined influence of both?)

The validation procedure used in the present paper aims to address these shortcomings. Validation does not focus on the model results but on its governing assumptions. Flow simulations are carried out over refined grids using a wide variety of building layouts and initial/boundary conditions. Since the purpose is to assess the ability of the models to account for urban anisotropy, the geometries are made intentionally strongly anisotropic, with strongly marked preferential directions. The refined simulation results, considered as an experimental reference, are used to compute domain- and boundary-based averages on the pore scale of the porosity model. The accuracy of the model is assessed by comparing the theoretical relationships between the domain- and boundary-based averages to the experimental 
ones. This approach has the advantage that (i) the errors in the fluxes and source terms are assessed separately, (ii) the reference data can be used to propose improved closure models, (iii) the analysis allows guidelines to be derived for the experimental, laboratory scale validation of fluxes and source term models. While experimental data sets would have been preferable, only refined flow simulations can be used used as reference solutions because the various laboratory experiments reported in the literature do not allow for the direct, systematic type of validation presented here.

The flow configurations explored in the present analysis are highly idealised. In particular, the building geometries are made strictly periodic so as to yield uniform porosity parameters and welldefined flux and source term closure relationships. For the same reason, the effects of bottom friction, turbulence and bottom slope are disregarded. As a result, only flat topographies are considered. This obviously is a limitation of the present work in that real-world urban geometries are non-periodic, the buildings are not exactly aligned, and the subgrid-scale topography may exert a very strong influence on the flow dynamics, especially when small depths are involved. All these factors may result in extra fluxes and source terms that are not accounted for in the present study. The interactions with urban drainage networks, that often occur during urban flods, are also neglected. However, analyzing idealised situations is deemed an indispensable prerequisite to applying porosity models to more complex flow configurations.

The present paper aims to answer the following questions:

Q1. How do the SP, IP and DIP models compare in terms of closure and source term model accuracy?

Q2. Does the connectivity porosity alone provide sufficient information to compute accurate fluxes and source terms, or are additional geometric descriptors needed?

Q3. How can the mass and/or momentum fluxes and source term closures in porosity models be further improved?

Q4. How can the results of the present analysis be used to design laboratory experiments (e.g. scale models of urban environments) for the experimental validation o porosity-based shallow water models?

The paper is structured as follows. Section 2 recalls the underlying assumptions and governing equations for the SP, IP and DIP models. The equations are presented in a uniform fashion for the sake of model comparison. Section 3 presents the simulation base. A simulation is defined as the combination of an urban geometry and an initial- or boundary-value problem. In this work, 96 simulations have been carried out on refined grids. Section 4 is devoted to the analysis of the flux closure formulae in the SP, IP and DIP models. Section 5 deals with the assessment of the source term models, including the transient momentum dissipation model [14] and the generalised tensor model [38]. Section 6 is devoted to a summary of results and a discussion. Conclusions are provided in Section 7.

\section{Models}

\subsection{Overview of porosity models}

The Single Porosity (SP), Integral Porosity (IP) and Dual Integral Porosity (DIP) models are obtained by averaging the two-dimensional shallow water equations over a domain $\Omega$ with boundary $\Gamma$. The main difference with the two-dimensional shallow water equations is that the domain $\Omega$ is only partially occupied by water, part of it being occupied by a solid phase (the buildings in the case of an urban area). Two types of porosity are defined: a domain-based (also called storage, or areal) porosity $\phi_{\Omega}$, that is the fraction of plan view area available to water storage, and a boundary (or conveyance, or connectivity) porosity $\phi_{\Gamma}$, that is the frontal area available for mass and momentum transfer. Both porosities are defined using a phase indicator $\epsilon(x, y)$, equal to unity if the point $(x, y)$ is in the liquid phase and that takes the value zero if $(x, y)$ belongs to the solid phase

$$
\phi_{\Omega}=\frac{1}{\Omega} \int_{\Omega} \epsilon(x, y) \mathrm{d} \Omega, \phi_{\Gamma}=\frac{1}{\Gamma} \int_{\Gamma} \epsilon(x, y) \mathrm{d} \Gamma
$$


The governing equations for the three models can be written in the general integral form

$$
\begin{gathered}
\partial_{t} \int_{\Omega} \mathbf{u} \mathrm{d} \Omega+\int_{\Gamma} \mathbf{F n} \mathrm{d} \Gamma=\int_{\Omega} \mathbf{s}_{\Omega} \mathrm{d} \Omega+\int_{\Gamma} \mathbf{s}_{\Gamma} \mathrm{d} \Gamma \\
\mathbf{u}=\phi_{\Omega}[h, q, r]^{T}=\phi_{\Omega}[h, h u, h v]^{T} \\
\mathbf{s}_{\Omega}=\phi_{\Omega}\left[0,\left(S_{0, x}-S_{f, x}\right) g h,\left(S_{0, y}-S_{f, y}\right) g h\right]
\end{gathered}
$$

where $\mathbf{u}$ is the conserved variable vector, $\mathbf{F}$ is the flux tensor, $\mathbf{n}$ is the normal outward unit vector to the boundary $\Gamma, \mathbf{s}_{\Omega}$ and $\mathbf{s}_{\Gamma}$ are respectively the domain- and boundary-based source terms, $h$ is the water depth, $q$ and $r$ are respectively the $x-$ and $y$-unit discharges, $u$ and $v$ are respectively the $x-$ and $y$-components of the flow velocity. The domain-based source term $\mathbf{s}_{\Omega}$ accounts for the horizontal forces arising from the bottom slope, the resistance exerted onto the flow by the solid phase (i.e. the buildings) and bottom friction. The boundary-based source term $\mathbf{s}_{\Gamma}$ accounts for the pressure force exerted by the solid-liquid interface. It includes for instance the effects of porosity gradients.

The dual nature of the porosity is extended to the flow variables. A boundary-based conserved variable $\mathbf{u}_{\Gamma}=\left[h_{\Gamma}, q_{\Gamma}, r_{\Gamma}\right]$ is defined in addition to the domain-based variable $\mathbf{u}$. The differences between the SP, IP and DIP models essentially result from different closure models between $\mathbf{u}$ and $\mathbf{u}_{\Gamma}$.

\subsection{Flux closure models}

SP model. In the SP model [15, 16, 37, the storage and connectivity porosities are assumed identical

$$
\begin{gathered}
\phi_{\Gamma}=\phi_{\Omega}=\phi,(h, q, r)_{\Gamma}^{T}=(h, q, r)^{T} \\
\mathbf{F}=\phi\left[\begin{array}{cc}
q & r \\
\frac{q^{2}}{h}+\frac{g}{2} h^{2} & \frac{q r}{h} \\
\frac{q r}{h} & \frac{r^{2}}{h}+\frac{g}{2} h^{2}
\end{array}\right]
\end{gathered}
$$

Integral Porosity (IP) model. The IP model [34] is derived considering that the storage and connectivity porosities are different in the general case. The domain and boundary variables are assumed identical

$$
\begin{gathered}
(h, q, r)_{\Gamma}^{T}=(h, q, r)^{T} \\
\mathbf{F}=\phi_{\Gamma}\left[\begin{array}{cc}
q & r \\
\frac{q^{2}}{h}+\frac{g}{2} h^{2} & \frac{q r}{h} \\
\frac{q r}{h} & \frac{r^{2}}{h}+\frac{g}{2} h^{2}
\end{array}\right]
\end{gathered}
$$

Dual Integral Porosity (DIP) model. As the IP model, the DIP model [14] uses $\phi_{\Omega} \neq \phi_{\Gamma}$, but the closure model is different. The boundary unit discharge is defined so as to preserve continuity across $\Gamma$

$$
\begin{gathered}
h_{\Gamma}=h,(q, r)_{\Gamma}^{T}=\frac{\phi_{\Omega}}{\phi_{\Gamma}}(q, r)^{T} \\
\mathbf{F}=\phi_{\Gamma}\left[\begin{array}{cc}
q_{\Gamma} & r_{\Gamma} \\
\frac{q_{\Gamma}^{2}}{h_{\Gamma}}+\frac{g}{2} h_{\Gamma}^{2} & \frac{q_{\Gamma} r_{\Gamma}}{h_{\Gamma}} \\
\frac{q_{\Gamma} r_{\Gamma}}{h_{\Gamma}} & \frac{r_{\Gamma}^{2}}{h_{\Gamma}}+\frac{g}{2} h_{\Gamma}^{2}
\end{array}\right]=\left[\begin{array}{cc}
\phi_{\Omega} q & \phi_{\Omega} r \\
\frac{\phi_{\Omega}^{2}}{\phi_{\Gamma}} \frac{q^{2}}{h}+\phi_{\Gamma} \frac{g}{2} h^{2} & \frac{\phi_{\Omega}^{2}}{\phi_{\Gamma}} \frac{q r}{h} \\
\frac{\phi_{\Omega}^{2}}{\phi_{\Gamma}} \frac{q r}{h} & \frac{\phi_{\Omega}^{2}}{\phi_{\Gamma}} \frac{r^{2}}{h}+\phi_{\Gamma} \frac{g}{2} h^{2}
\end{array}\right]
\end{gathered}
$$




\subsection{Source term models}

The purpose is to test expressions for the vector

$$
\mathbf{s}_{f}=\left[S_{f, x}, S_{f, y}\right]^{T}
$$

Early versions of the SP model [15, 16, 37] and the IP model [34] use an isotropic energy loss formula in the form

$$
\mathbf{s}_{f}=k_{I}|\mathbf{v}| \mathbf{v}
$$

where $k_{I}$ is a coefficient that incorporates the lumped effects of bottom friction and building resistance. In [15, a Borda-type head loss model was proposed. It was later invalidated by detailed inspection of flow simulation results over refined grids [11]. In [34, building and bottom drag coefficients were proposed as an alternative to more widespread Chezy-Manning-Strickler friction laws.

In [14], test simulations involving the propagation of positive and negative waves in idealized networks of orthogonal streets are reported. The simulations indicate that, in the absence of bottom friction, the momentum source term should be split into two parts as

$$
\mathbf{s}_{f}=\mathbf{s}_{T}+\mathbf{s}_{D}
$$

where $\mathbf{s}_{T}$ and $\mathbf{s}_{D}$ are respectively a transient and a drag source term. The models for these two source terms are examined in the following two paragraphs.

Transient momentum dissipation model. The source term $\mathbf{s}_{T}$ accounts for a momentum dissipation mechanism that cannot be accounted for by usual, friction-based head loss models [14]. It is active only under transient conditions involving positive waves (rising water levels). It stems from the dissipation of moving bores due to the reflection against building walls. When steady state is reached, or when the water level decreases, no momentum dissipation occurs due to shock reflection and $\mathbf{s}_{T}$ is zero. This dissipation mechanism is essential to an accurate reconstruction of water level and unit discharge fields across shocks. The following form is shown to preserve the observed self-similarity properties of the flow solution [14]:

$$
\mathbf{s}_{T}=\left\{\begin{array}{cc}
{\left[\begin{array}{cc}
\mu_{x x} & \mu_{x y} \\
\mu_{y x} & \mu_{y y}
\end{array}\right] \nabla \cdot \mathbf{F}_{m}} & \text { if } \quad \partial_{t} h>0 \\
0 & \text { if } \quad \partial_{t} h \leq 0
\end{array} \quad, \quad \mathbf{F}_{m}=\phi_{\Gamma}\left[\begin{array}{cc}
\frac{q^{2}}{h}+\frac{g}{2} h^{2} & \frac{q r}{h} \\
\frac{q r}{h} & \frac{r^{2}}{h}+\frac{g}{2} h^{2}
\end{array}\right]\right.
$$

where $\nabla . \mathbf{F}_{m}$ denotes the divergence of the momentum flux tensor $\mathbf{F}_{m}$. So far, no satisfactory model has been proposed for the dissipation terms. Consequently, the tensor coefficients $\mu_{i j}$ must be calibrated.

Building drag dissipation model. The source term $\mathbf{s}_{D}$ accounts for building drag forces. The dynamics of free surface flow in three and four branch crossroads has been studied extensively over the past years, from both an experimental and numerical point of view $17,9,10,17,22,23,24,25$, 27, 28, 31, 32, 33. These studies have mainly focused on the flow distribution between the outflowing branches of the crossroads (see e.g. [25, for the subcritical case, [33, for transcritical regimes, [32] for the subcritical case, [17, for a numerical study). The empirical relationships derived in these studies cannot be used as such to infer source term formulae in two-dimensional porosity models, for the following reasons. Firstly, the studies focus on the flow distribution between the branches. Additional measurements (such as the distribution of the velocity field and free surface elevation across each of the four branches) would be needed to infer the drag coefficient from a two-dimensional momentum balance. Secondly, the downstream boundary conditions are reported to have a strong influence on the flow patterns [33]. The boundary conditions used in many experiments (e.g. presence of downstream weirs for flow measurements) are not compatible with the flow conditions met with series of connected crossroads as considered in the present study. Thirdly, the purpose of the present study is to assess upscaled model of the two-dimensional shallow water equations. Neither viscous nor turbulent head losses are accounted for in the refined flow simulations reported here, while they probably play an important role in the reported experiments. For all these reasons, the abovementioned studies are not used in the present work. 
Energy loss models specific to the shallow water models with porosity have been proposed in the literature. In [15, 34, 37] an isotropic head loss model is used. Clearly, such a model is not adapted to the strongly anisotropic flow patterns occurring in urban areas. Two anisotropic models have been proposed recently. A tensor formulation is proposed in [14]:

$$
\mathbf{s}_{D}=-\mathbf{C}_{D}|\mathbf{v}| \mathbf{v}
$$

where $\mathbf{C}_{D}$ and $\mathbf{v}$ are respectively a drag tensor and the velocity vector. A more general model is proposed in [38] for orthogonal street networks:

$$
\mathbf{s}_{D}=-(1+a|\sin 2 \theta|) \mathbf{C}_{D}|\mathbf{v}| \mathbf{v}
$$

where $a$ is a positive, dimensionless coefficient and $\theta$ is the angle between the velocity vector and the main street axis. The first model requires that the drag tensor $\mathbf{C}_{D}$ be calibrated, while the second provides one more degree of freedom with the calibration of $a$. It is worth noting that all previously published drag models can be rewritten as particular cases of the model (11).

All three models $(7,10,11)$ are deemed incomplete in that they fail to represent an essential flow feature. Consider a periodic layout formed by square building blocks with equal spacing in the E-W and N-S streets. Under the assumption of a frictionless, horizontal bottom, a uniform flow velocity field aligned with any of the two orthogonal main street directions $\left(\theta=0\right.$ or $\left.\frac{\pi}{2}\right)$ yields a horizontal free surface and a zero drag force. This yields a zero $k_{f}$ for the model (7) and a zero drag tensor for the two models (10 11). Consequently, $\mathbf{s}_{D}$ is zero regardless of the magnitude and direction of the velocity field $\mathbf{v}$, including in the configuration $\theta=\frac{\pi}{4}$, where it is known from experiments to be maximum [38] To overcome this problem, it is proposed that the formula (11) be revised as follows

$$
\mathbf{s}_{D}=-|\sin 2 \theta| \mathbf{C}_{D}|\mathbf{v}| \mathbf{v}
$$

This model yields a zero drag force for $\theta=0$ and $\theta=\frac{\pi}{2}$, while $\theta= \pm \frac{\pi}{4}$ yields a maximum drag, as expected from [38. The proposed model can be viewed as a limit case of Velickovic et al's model as $a$ tends to infinity in equation (11). The tensor $\mathbf{C}_{D}$ in equation 12 is then equal to the product $a \mathbf{C}_{D}$ in equation (11).

It must be noted that the building drag models 11,12 are valid only for square building layouts, that is $L_{1}=L_{2}$ and $W_{1}=W_{2}$. As a matter of fact, a key assumption to these two models is that the the maximum drag occurs for $\theta= \pm \frac{\pi}{4}$. This is obviously not the case when the building geometry is not the same in the $x$ - and $y$-directions. In such a case, it would be necessary to introduce additional parameters in the model to indicate the directions of maximum drag. Besides, it is not certain that such directions would be orthogonal and that the tensor descriptions (11) 12 would remain meaningful. For this reason, only square layouts are explored in the analysis of the building drag model.

\section{Simulation base}

\subsection{Model geometries}

Model geometry is based on a periodic building layout. In order to allow for accurate statistics and to assess the self-similarity properties of the solutions, the number of periods involved is very large (more than 100 in most simulations). The layout is generated by two series of regularly spaced, parallel streets. The angle between the two street directions is denoted by $\alpha$ (see Figure 1). By assumption, the first street direction is aligned with the $x$-axis. 

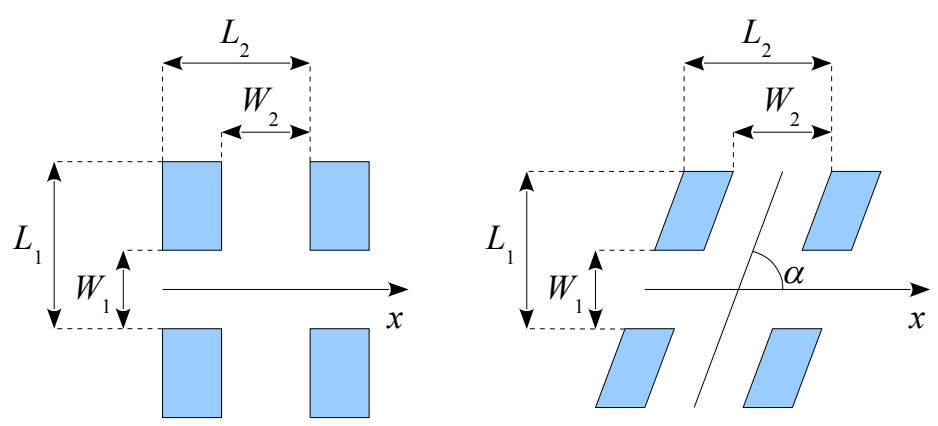

Figure 1: Model geometry. Definition sketch.

The $p$ th street network $(p=1,2)$ yields a frontal porosity $\phi_{p}=\frac{W_{k}}{L_{k}}$, where $L_{k}$ and $W_{k}$ are respectively the spacing and width of the streets. The domain porosity $\phi_{\Omega}$, defined as the fraction of plan view area available for water storage, is thus $\phi_{\Omega}=\phi_{1}+\phi_{2}-\phi_{1} \phi_{2}$. In this work, nine different geometries are considered (Table 1).

\begin{tabular}{ccccccccc}
\hline Model name & $L_{1}(\mathrm{~m})$ & $W_{1}(\mathrm{~m})$ & $L_{2}(\mathrm{~m})$ & $W_{2}(\mathrm{~m})$ & $\alpha(\mathrm{rad})$ & $\phi_{\Omega}$ & $\phi_{1}$ & $\phi_{2}$ \\
\hline M1a & 75 & 25 & 150 & 50 & $\pi / 2$ & $5 / 9$ & $1 / 3$ & $1 / 3$ \\
M1b & 150 & 50 & 150 & 50 & $\pi / 2$ & $5 / 9$ & $1 / 3$ & $1 / 3$ \\
M1c & 150 & 50 & 75 & 25 & $\pi / 2$ & $5 / 9$ & $1 / 3$ & $1 / 3$ \\
M2 & 100 & 20 & 100 & 20 & $\pi / 2$ & $9 / 25$ & $1 / 5$ & $1 / 5$ \\
M3 & 100 & 10 & 100 & 10 & $\pi / 2$ & $19 / 100$ & $1 / 10$ & $1 / 10$ \\
M4 & 100 & 20 & 50 & 20 & $\pi / 2$ & $13 / 25$ & $1 / 5$ & $2 / 5$ \\
M5 & 50 & 20 & 100 & 20 & $\pi / 2$ & $13 / 25$ & $2 / 5$ & $1 / 5$ \\
M6 & 150 & 50 & 150 & 50 & $\tan ^{-1}(2)$ & $5 / 9$ & $1 / 3$ & $1 / 3$ \\
M7 & 150 & 50 & 150 & 50 & $\pi / 4$ & $5 / 9$ & $1 / 3$ & $1 / 3$ \\
\hline
\end{tabular}

Table 1: Model nomenclature. Notation: see Figure 1.

Geometries M1a, b,c correspond to identical $\left(\alpha, \phi_{1}, \phi_{2}, \phi_{\Omega}\right)$ combinations, but these are obtained for different street widths and spacings. Model M1b is strictly isotropic, with $\left(L_{1}, W_{1}\right)=\left(L_{2}, W_{2}\right)$, while Models M1a and M1c are based on $\left(L_{1}, W_{1}\right)=\frac{1}{2}\left(L_{2}, W_{2}\right)$ and $\left(L_{1}, W_{1}\right)=2\left(L_{2}, W_{2}\right)$ respectively. The purpose is to assess whether the frontal porosity alone is a sufficient descriptor of the geometry. In Models M2 and M3, $\phi_{1}=\phi_{2}$ but these values are respectively $5 / 3$ and $10 / 3$ times as small as those in M1b. Comparing M2 and M3 to M1b allows the influence of the street width-to-spacing ratio to be assessed. Models M6 and M7 differ from the previous five in that the street angle $\alpha$ is not $\pi / 2$. While an intersection angle $\alpha=\tan ^{-1} 2$ (that is, approximately 63 degrees) may be found occasionally in real-world street networks (model M6), $\alpha=\pi / 4$ (model M7) is introduced with the purpose of testing extreme configurations. With Models M4 and M5, $\phi_{1} \neq \phi_{2}$, which is typical of a number of suburban layouts.

All models have a totally flat bottom. This assumption clearly introduces limitations. However, the main purpose here is to assess the accuracy of the porosity-based description of connectivity and storage properties of the urban medium, all other factors being disregarded.

All models are meshed using $1 \mathrm{~m}$ to $2.5 \mathrm{~m}$ wide cells. The objective is to discretize the geometry using 20 cells across the streets in as many cases as possible, so as to obtain a sufficiently accurate description of the hydraulic fields. To give but one example, with more than 100 street periods in the $x$-direction, Model M1b counts 212,000 quadrangular cells and 222,621 nodes. The two-dimensional shallow water equations are solved using the MUSCL-EVR technique [35. 


\subsection{Initial Value Problems (IVPs)}

The initial value problems are Riemann problems based on the so-called urban dambreak problem introduced in [11]. The water is initially at rest, with water depths $h_{L}$ and $h_{R}$ on the left- and righthand sides of a straight line passing at $(0,0)$. In a number of simulations, the interface between the left and right states is not perpendicular to the $x$-direction. Its normal unit vector makes an angle $\beta$ with the $x$-axis (Figure 2 left). Table 2 gives the characteristics of the various Riemann problems used in the present work. When $\beta \neq 0$, the transient propagation is oblique. Computational time is saved to a large extent by meshing only one street period in the $y$-direction and prescribing periodic boundary conditions [3, 21] on the Northern and Southern boundaries of the streets (Figure 2 right). In the example of Figure 2 sketched for a square layout, connecting the Northern boundary of a street to the Southern boundary of the next one (A with A', B with B', etc.) allows a 45 degrees propagation pattern to be obtained with one period only. An angle $\beta=\tan ^{-1}\left(\frac{1}{2}\right)$ is obtained by meshing two $x$-streets instead of a single one.

\begin{tabular}{cccc}
\hline RP name & $h_{L}(\mathrm{~m})$ & $h_{R}(\mathrm{~m})$ & $\beta(\mathrm{rad})$ \\
\hline R01 & 1.01 & 1 & 0 \\
R02 & 2 & 1 & 0 \\
R03 & 5 & 1 & 0 \\
R04 & 10 & 1 & 0 \\
R05 & 20 & 1 & 0 \\
R06 & 50 & 1 & 0 \\
R07 & 1.01 & 1 & $\pi / 4$ \\
R08 & 2 & 1 & $\pi / 4$ \\
R09 & 5 & 1 & $\pi / 4$ \\
R10 & 10 & 1 & $\pi / 4$ \\
R11 & 20 & 1 & $\pi / 4$ \\
R12 & 50 & 1 & $\pi / 4$ \\
R13 & 1.01 & 1 & $\tan ^{-1}(1 / 2)$ \\
R14 & 2 & 1 & $\tan ^{-1}(1 / 2)$ \\
R15 & 5 & 1 & $\tan ^{-1}(1 / 2)$ \\
R16 & 10 & 1 & $\tan ^{-1}(1 / 2)$ \\
R17 & 20 & 1 & $\tan ^{-1}(1 / 2)$ \\
R18 & 50 & 1 & $\tan ^{-1}(1 / 2)$ \\
\hline
\end{tabular}

Table 2: Initial Value Problem (IVP) nomenclature. Notation: see Figure 2. 

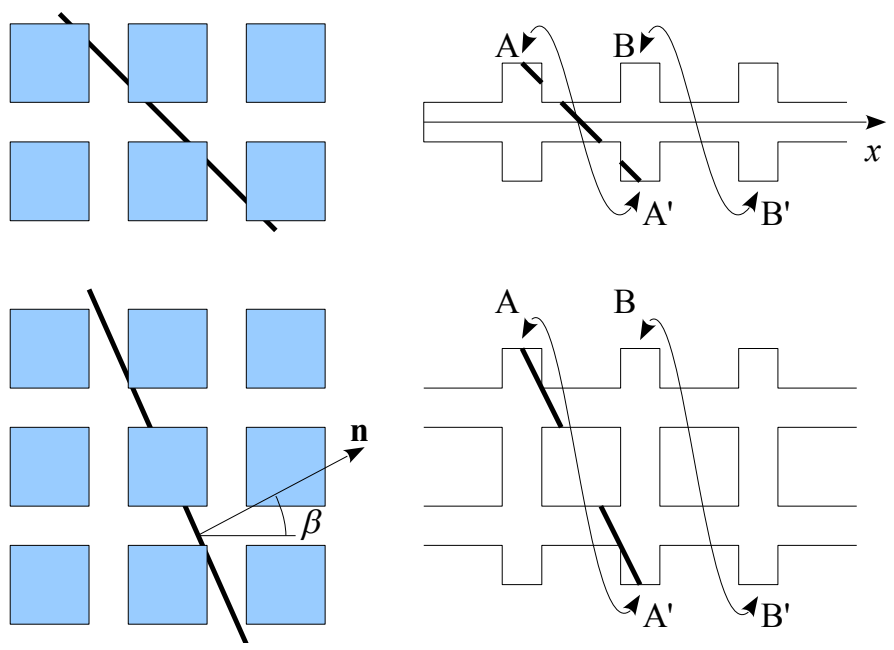

Figure 2: Defining oblique Riemann problems using periodic boundary conditions. Top: IVPs R07R12. Bottom: IVPs R13-R18.

\subsection{Boundary Value Problems (BVPs)}

The boundary value problems are used to assess the momentum source term models. The steady state solution of the following problem is sought. A square domain of size $L \times L$ corresponding to geometries M01-M7 is defined. The Western and Southern boundaries are split into two parts of equal length (Figure 3). The half-side extending from the South-Western corner is assigned a prescribed water level $h_{1}$, while the remaining half of the Western and Southern edges are assigned an impervious condition. The Eastern and Northern boundaries are assigned a prescribed water level $h_{2}$. Table 3 gives the combinations $\left(h_{1}, h_{2}\right)$ used for the various BVPs. The transient simulation is started from a uniform initial condition $\mathbf{u}(x, y, 0)=\left[h_{0}, 0,0\right]^{T} \forall(x, y) \in[0, L] \times[0, L]$ until steady state is reached. For $L=1800 \mathrm{~m}$, steady state is achieved at $t=10^{4} \mathrm{~s}$.

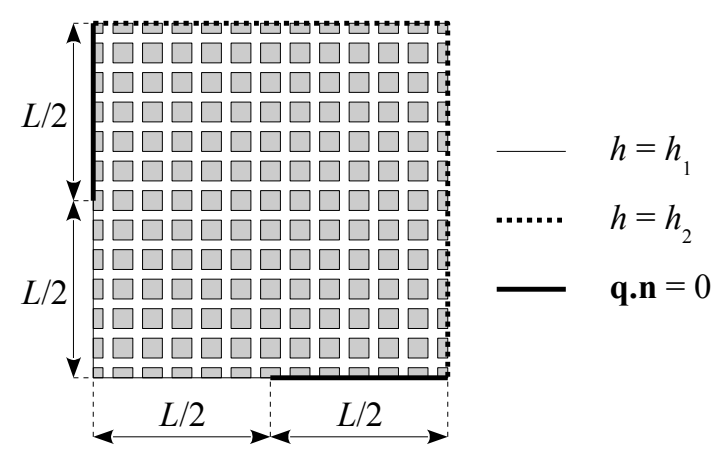

Figure 3: Boundary Value Problems. Boundary condition definition sketch. 


\begin{tabular}{ccc}
\hline IVP name & $h_{1}(\mathrm{~m})$ & $h_{2}(\mathrm{~m})$ \\
\hline $\mathrm{B} 01$ & 1.01 & 1 \\
$\mathrm{~B} 02$ & 2 & 1 \\
$\mathrm{~B} 03$ & 5 & 1 \\
$\mathrm{~B} 04$ & 10 & 1 \\
$\mathrm{~B} 05$ & 20 & 1 \\
$\mathrm{~B} 06$ & 50 & 1 \\
\hline
\end{tabular}

Table 3: Boundary value problem (BVP) nomenclature. Notation: see Figure 3.

\section{Flux model analysis}

\subsection{Method}

The accuracy of the SP, IP and DIP flux closure models is assessed using the numerical results of IVPs R01-R18. A cross-section (called interface in what follows) $\Gamma$ is defined between two adjacent buildings, as shown in Figure 4. The interface may be normal to the $x$ - or $y$-direction. An averaging volume $\Omega$ is centred around the interface. $\Omega$ extends over one spatial period on each side of the interface. The various flow variables and components of the flux tensor are averaged over $\Gamma$ and $\Omega$, in agreement with the approach developed in [19].
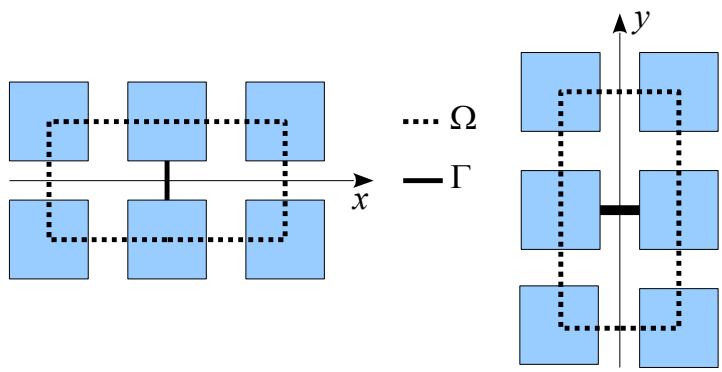

Figure 4: Flux closure model analysis. Definition sketch. Left: $x$-facing interface $\Gamma$. Right: $y$-facing interface $\Gamma$.

The analysis consists in plotting the interface-averaged variables and fluxes versus the domainaveraged ones and comparing these averages to the theoretical closure relationships (3a $5 \mathrm{~b})$. Table 4 gives the relationships derived from the SP, IP and DIP closure models. For each simulation, two interfaces $\Gamma$ with their surrounding domains $\Omega$ are chosen: one in the rarefaction wave of the Riemann problem, the other in the shock wave. The purpose is to check the validity of the closure relationships for both continuous and discontinuous flow patterns.

\begin{tabular}{cccccccc}
\hline Model & $h_{\Gamma}$ & $q_{\Gamma}$ & $r_{\Gamma}$ & $\left(\frac{q^{2}}{h}\right)_{\Gamma}$ & $\left(\frac{q r}{h}\right)_{\Gamma}$ & $\left(\frac{r^{2}}{h}\right)_{\Gamma}$ & $\left(h^{2}\right)_{\Gamma}$ \\
\hline SP & $h_{\Omega}$ & $q_{\Omega}$ & $r_{\Omega}$ & $\frac{q_{\Omega}^{2}}{h_{\Omega}}$ & $\frac{q_{\Omega} r_{\Omega}}{h_{\Omega}}$ & $\frac{r_{\Omega}^{2}}{h_{\Omega}}$ & $\left(h_{\Omega}\right)^{2}$ \\
IP & $h_{\Omega}$ & $q_{\Omega}$ & $r_{\Omega}$ & $\frac{q_{\Omega}^{2}}{h_{\Omega}}$ & $\frac{q_{\Omega} r_{\Omega}}{h_{\Omega}}$ & $\frac{r_{\Omega}^{2}}{h_{\Omega}}$ & $\left(h_{\Omega}\right)^{2}$ \\
$\mathrm{DIP}$ & $h_{\Omega}$ & $\frac{\phi_{\Omega}}{\phi_{\Gamma}} q_{\Omega}$ & $\frac{\phi_{\Omega}}{\phi_{\Gamma}} r_{\Omega}$ & $\left(\frac{\phi_{\Omega}}{\phi_{\Gamma}}\right)^{2} \frac{q_{\Omega}^{2}}{h_{\Omega}}$ & $\left(\frac{\phi_{\Omega}}{\phi_{\Gamma}}\right)^{2} \frac{q_{\Omega} r_{\Omega}}{h_{\Omega}}$ & $\left(\frac{\phi_{\Omega}}{\phi_{\Gamma}}\right)^{2} \frac{r_{\Omega}^{2}}{h_{\Omega}}$ & $\left(h_{\Omega}\right)^{2}$ \\
\hline
\end{tabular}

Table 4: Relationships between the interface- and domain-averaged variables and flux components for the SP, IP and DIP models. The subscript $(\Gamma$ or $\Omega$ ) indicates the averaging domain. 


\subsection{Mass flux closure}

The mass flux is $\mathbf{q}_{\Gamma} \cdot \mathbf{n}$. It is equal to $q_{\Gamma}$ over $x$-facing interfaces and equal to $r_{\Gamma}$ over $y$ - facing interfaces. For the sake of paper readability, the graphs illustrating the analysis are sent to Appendix A.1. Two types of behaviours are observed .

The first type of behaviour is found for the combinations of Models M1-5 and IVPs R01-R06. The DIP closure is observed to be accurate for both $q_{\Gamma}$ and $r_{\Gamma}$ (Figures 12 and 13), while the SP and IP closures yield underestimated $q_{\Gamma}$ values. Given the symmetry of the problem, $r_{\Gamma}$ is zero regardless of the closure used. It is also zero for $y$-facing interfaces (as predicted by all closure models), which is why no graph is displayed for these interfaces.

A second type of behaviour is encountered for Models M1 and M4 with IVPs R07-18, as well as models M6-M7 with IVPs R01-R06. A common feature to these simulations is that the direction of the Riemann problem is oblique to at least one of the principal directions of the street network. Figures 14 and 15 show respectively the scatter plots for $\left(q_{\Omega}, q_{\Gamma}\right)$ and $\left(r_{\Omega}, r_{\Gamma}\right)$ obtained from IVPs R07R18 and Model M1b. Similar behaviours are observed for model M4 but are not shown here for the

sake of paper conciseness. The normal component of the unit discharge $\left(q_{\Gamma}\right.$ for $x$-facing interfaces on Figure 14, $r_{\Gamma}$ for $y$-facing interfaces on Figure 15) is predicted accurately by the DIP closure and underestimated by the SP-IP closures. All closures, however, fail dramatically in estimating the tangent component of the unit discharge $\left(r_{\Gamma}\right.$ for $x$-facing interfaces on Figure $14 q_{\Gamma}$ for $y$-facing interfaces on Figure 15). As a matter of fact, all tangent components over $\Gamma$ are zero, while the closures predict non-zero values. Similar trends are observed for Models M6-7 with IVPs R01-06 (Figures 16 and 17): the normal components ( $q_{\Gamma}$ along $x$-facing interfaces and $r_{\Gamma}$ along $y$-facing interfaces) are predicted rather accurately by the DIP closure, while the tangent components ( $r_{\Gamma}$ along $x$-facing interfaces and $q_{\Gamma}$ along $y$-facing interfaces) are wrongly predicted by all closure models. Examining the unit discharge fields allows this failure to be explained. As an example, the unit discharge vector field simulated at $t=1000$ s for Simulation M1b-R10 are displayed on Figure 5 . The vector field clearly exhibits three zones: the crossroads with vectors making roughly a 45 degree angle with the main street axes, the E-W street with vectors parallel to the $x$-axis and the N-S street with vectors parallel to the $y$-axis. Similar patterns are observed with non-orthogonal street networks (M6-7). The flow field in the streets is strongly aligned with the street main axis. Building layout forcing the flow field along preferential directions has been observed in simulations involving much sparser building layouts than those explored in the present study [14.

To summarize, only the DIP closure yields a correct estimate for the normal component of the unit discharge. All closures give erroneous estimates for the transverse unit discharge when the flow is not aligned with the main street directions. This, however, impacts the mass flux in a very limited fashion because only the normal unit discharge is involved in the mass balance. 


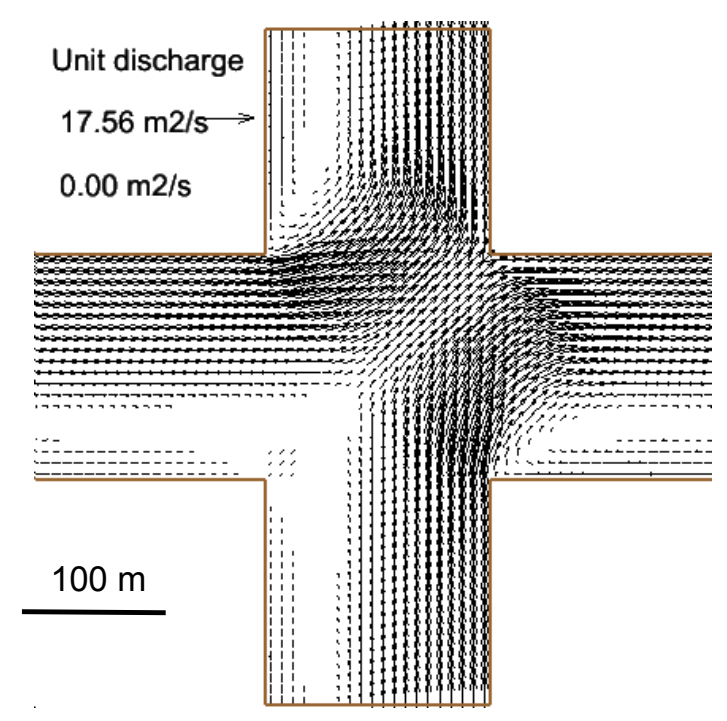

Figure 5: Simulation M1b-R10. Unit discharge field at $t=1000 \mathrm{~s}$ for $x \in[300 \mathrm{~m}, 450 \mathrm{~m}]$.

\subsection{Momentum flux closure}

The momentum flux tensor involves the hydrostatic pressure term $\frac{g}{2} h^{2}$ and the momentum discharges $\frac{q^{2}}{h}, \frac{q r}{h}, \frac{r^{2}}{h}$.

The SP, IP and DIP water depth closures are identical. This results in identical closure relationships for the pressure term in the three models. Figure 6 shows the scatter plots obtained for all simulations (models M1-7, IVPs R01-18). The SP, IP and DIP closures are observed to agree extremely well with the simulation results.
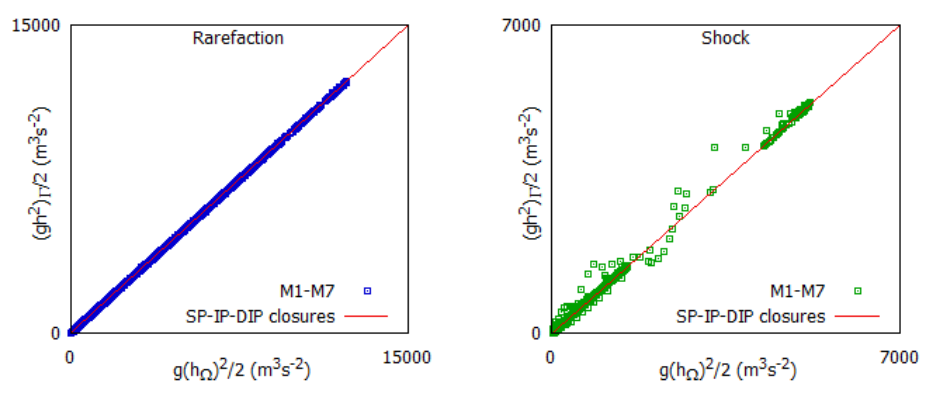

Figure 6: All simulations. Scatter plot for the hydrostatic pressure term.

The momentum flux closures follow the same trends as the mass flux closures. When the main two street directions are orthogonal and the Riemann problem is along one of these two directions, the DIP closure gives very good estimates for the momentum discharge $\frac{q^{2}}{h}$ (see Appendix A.2, Figure 18. The SP and IP closures yield strongly underestimated values for $\frac{q^{2}}{h}$. Only two types of (limited) inaccuracy are observed for the DIP closure. The first is observed on Figure 18, In the rarefaction wave, the normal momentum flux $q^{2} / h$ is slightly larger than the value predicted by the DIP closure. This is because the unit discharge field is not uniform across the longitudinal streets. This effect has been identified and explained in [14] as follows. In the rarefaction wave, the dropping water level causes the water to flow from the lateral streets into the main street. This water has a zero $x$-velocity. The mixing with the water flowing in the $x$-direction result in a low-velocity layer near the walls. Since $q$ is not uniform over the cross-section, the average of $q^{2}$ is necessarily larger than the square of the average $q$. Note that this effect is not observed in the shock wave. The second type of inaccuracy 
is observed for the closures $q r / h$ and $r^{2} / h$ (Figures 19 and 20) across $x$-facing interfaces. While all closures would theoretically predict zero values owing to problem symmetry, a non-zero average $q r / h$ is observed for Model M4 (Figure 19) and the average $r^{2} / h$ is consistently non-zero (Figure 20). It must be noted, however, that these non-zero values are several orders of magnitude smaller than those of $q^{2} / h$. Moreover, erroneous $r^{2} / h$ values over $x$-facing interfaces have no consequence on momentum balance because $r^{2} / h$ contributes to the momentum balance only over $y$-facing interfaces.

When the Riemann problem is not aligned with the main street directions, all closures yield strongly inaccurate estimates. This is illustrated by Figure 21. where the average momentum flux $q^{2} / h$ is displayed for Model M1b with IVPs R07-18. As shown in Figure 5, the unit discharge field is strongly non-uniform across both E-W and N-S streets. It is also collinear to the street axes. This results in (i) all closure models underestimating the average $q^{2} / 2$ over $x$-facing interfaces (Figure 21, left) and (ii) identically zero values across $y$-facing interfaces when all closure models predict non-zero values (Figure 21 right). Similar errors are observed for Models M6-7 with IVPs R01-06 (Figure 22).

In all simulations, however, the DIP closure is consistently closer to the computed average values than are the SP and IP closures. An accurate momentum flux closure should incorporate two corrections. The first is that the boundary unit discharge field should be aligned with the street axis, the second is that the normal momentum flux $q^{2} / h$ be replaced with $\chi q^{2} / h$, where $\chi \geq 1$ is a correction factor accounting for the non-uniform distribution of $q$ across the interface. The non-uniform flow velocity field across the street section was mentioned in [14. It is visible from Figure 21 (left) that $\chi$ is little sensitive to the magnitude of the velocity (R07-R12 yield very similar correction factors, just as R13-18 do) and that it is a function of the angle $\beta$ between the main street axis and the direction of the Riemann problem. The average $\chi$ for IVPs R07-12 $\left(\beta=\frac{\pi}{4}\right.$, Figure 21 upper left $)$ is slightly larger than 2, while it is only 1.1 for IVPs R13-18 $\left(\beta=\tan ^{-1}\left(\frac{1}{2}\right)\right.$, Figure 21 lower left). Proposing accurate $\chi(\beta)$ functions, however, is beyond the scope of the present paper.

\section{$5 \quad$ Source term model analysis}

\subsection{Self-similarity of Riemann Problem solutions}

Numerical experiments indicate that a positive wave propagating into a periodic, orthogonal street network along one of the main street directions generates momentum dissipation, while a negative wave does not [14]. Moreover, the source term is known to be zero for steady state flow parallel to one of the the main street directions [14, 38. Consequently, the momentum dissipation mechanism does not obey an equation of state (it is not a function of the flow solution alone). A specific momentum dissipation model was proposed for the MP model [11] and the recently published DIP model [14] to account for this. In this model, the momentum source term $\mathbf{s}_{T}$ is a linear function of the divergence of the momentum flux. Incorporating this source term into the governing equations yields a homogeneous hyperbolic system of conservation laws. A salient feature of such a system is that the solutions of the Riemann problem are self-similar in $(x, t)$ [20]. The solution depends only on the ratio $x / t$, where $x$ is the abscissa counted from the location of the initial discontinuity. If the hyperbolic system is non-homogeneous (which is the case when the source term obeys an equation of state), the solution is not self-similar. Testing the self-similarity of the Riemann problem solutions is an efficient way of discriminating between two source terms with different structures.

The self-similarity of the solutions is checked for all the IVPs (M1-7, R01-06). Four main flow configurations are observed. They are illustrated by Figure 7. On the figure, four typical normalized water depth maps in the $(x, t)$ plane are displayed. The normalized water depth $h^{*}$ is defined as

$$
h^{*}=\frac{h-h_{R}}{h_{L}-h_{R}}
$$

It is equal to zero and unity for $h=h_{R}$ and $h=h_{L}$ respectively. The maps displayed on Figure 7 are obtained by averaging the flow solution over a building period $L_{2} \times L_{1}$. The 42 normalized water depth maps are shown in the Appendix (Figure 23) and will not be detailed here for the sake of conciseness.

The first type of behaviour is observed for simulations involving orthogonal street directions $\left(\alpha=\frac{\pi}{2}\right)$ and Riemann problems propagating along one of the two main street directions $(\beta=0)$. 42 such 
simulations have been carried out for the present work (the 7 Model geometries M1-M5 combined with the 6 IVPs R01-06). This behaviour is illustrated by Figure $7 \mathrm{a}$. The contour lines for $h^{*}$ are straight lines in the $(x, t)$ plane, indicating that the solution depends on the ratio $x / t$ alone. A similar behaviour is observed for the unit discharge maps (not shown here for the sake of conciseness). It is observed for 37 simulations out of the total 42 .

A limited number of configurations $(\alpha, \beta)=\left(\frac{\pi}{2}, 0\right)$ lead to a somewhat different behaviour, as illustrated by Figure77. In Simulations M1b-R04, M3-R04, M4-R02, M4-R03 and M5-R04, early times show a self-similar behaviour. After a time ranging from 100s to 500s depending on the simulations, a perturbation develops downstream of the initial discontinuity location. After a time ranging from $500 \mathrm{~s}$ to $1000 \mathrm{~s}$ depending on the simulations, the contour lines delineating this perturbation tend to follow straight lines in the $(x, t)$ plane. This seems to indicate the emergence of a new, self-similar solution at later times. The reason for this is unclear at the moment. Complementary simulations (not reported in the present paper) show that modifying the left and/or right states $h_{L}$ and $h_{R}$ by $10 \%$ prevents the perturbation from developing and the solution remains fully self-similar, as on Figure $7 \mathrm{a}$. The appearance of the perturbation may be attributed to a very specific combination of model geometry, flow velocities and water depths that may induce e.g. resonance conditions. The reader's attention is drawn to the following points: (i) the developing perturbation seems to impact only the intermediate region of constant state and not to impact the rarefaction wave on the left-hand side of $x=0$, (ii) the length of the perturbation (Figure $7 \mathrm{~b}$ ) at $t=1000 \mathrm{~s}$ is approximately 2000 metres (16 building blocks). The emergence of the perturbation could not have been detected from simulations involving only a few building periods.

The third an fourth main configuration types are respectively $\left(\alpha=\frac{\pi}{2}, \beta \neq 0\right)$ (see Figure 7 ) and $\alpha \neq \frac{\pi}{2}$ (Figure $7 \mathrm{~d}$ ). In both configurations, the self-similar character of the flow solution is broken. While the contour lines for $h^{*}$ seem to follow a linear trend away from the heads of the rarefaction and shock waves, the straight lines do not converge toward the point $(0,0)$ in the $(x, t)$ plane. Therefore, the solution is not a function of the ratio $x / t$ alone.

As a conclusion, while Riemann problems propagating along one of the main directions in orthogonal street networks yield self-similar solutions, other configurations do not. Checking the validity of the transient momentum dissipation model [14] is thus meaningful only for simulations $(\alpha, \beta)=\left(\frac{\pi}{2}, 0\right)$, that is, Models M1-5 with IVPs R01-06. This does not mean that this model is invalid in other flow configurations. However, for $\alpha \neq \frac{\pi}{2}$ and/or $\beta \neq 0$, its effects are clearly overridden by those of larger momentum source terms with a different nature. This is confirmed by the analysis in the next subsection. 
(a)

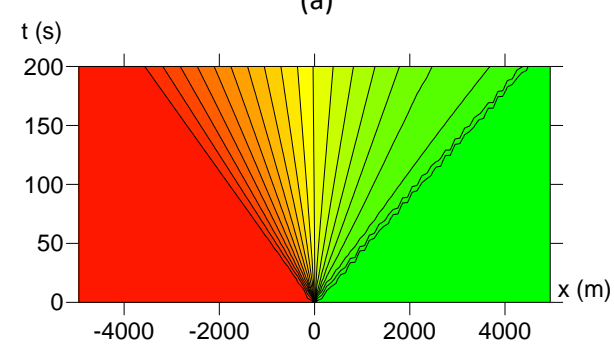

(c)

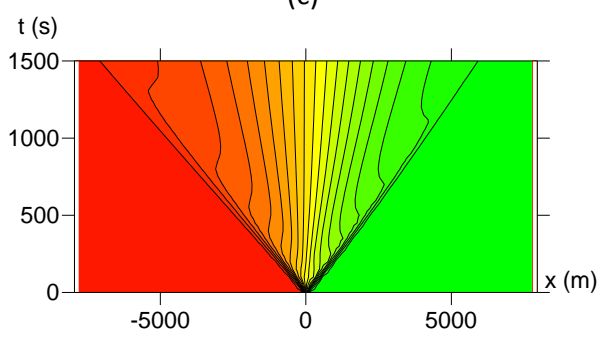

(b)

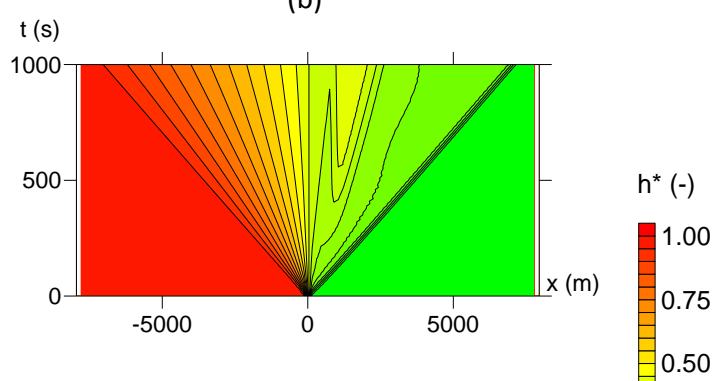

(d)

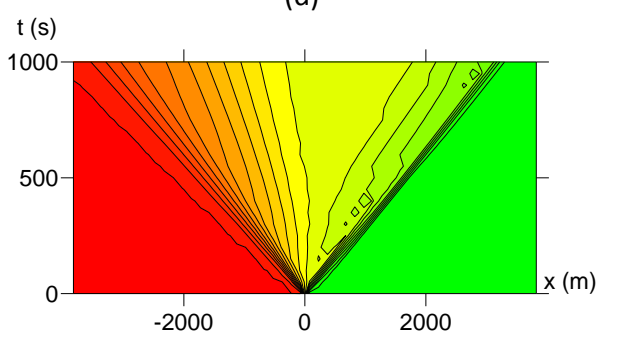

Figure 7: The four typical behaviours for the normalised water depth maps in the $(x, t)$ plane for various simulations. (a) Simulation M5-R06, (b) Simulation M1b-R04, (c) Simulation M1b-R08, (d) Simulation M6-R02. The small triangular contour lines on the right-hand side of the shock wave are graphical interpolation artefacts.

\subsection{Transient momentum dissipation model}

Bearing in mind the analysis of Subsection 5.1, the validity of the model $(9)$ is checked only for the simulations with a clearly identified self-similar behaviour. Models M1-M5 combined with IVPs R01R06 are analysed. The principle of the analysis is the following. At a given time $t$, the $x$-momentum equation is integrated with respect to space over the length of the simulation domain. It is assumed that $t$ is such that neither the rarefaction wave nor the shock wave have reached the boundaries of the computational domain. In this case, the flow velocity is zero at both ends of the domain $(x= \pm L)$ and the momentum flux at the boundary simplifies to $\phi_{\Gamma} \frac{g}{2} h^{2}$. The momentum equation in the $x$-direction is

$$
\partial_{t}\left(\phi_{\Omega} q\right)+\left(1-\mu_{x x}\right) \partial_{x} \phi_{\Omega}\left(\frac{q_{\Gamma}^{2}}{h_{\Gamma}}+\frac{g}{2} h_{\Gamma}^{2}\right)=0
$$

Integrating this equation over $[-L,+L]$, using the closure $h_{\Gamma}=h$ yields

$$
\partial_{t} \int_{-L}^{+L} \phi_{\Omega} q \mathrm{~d} x=\left(1-\mu_{x x}\right)\left[\frac{q_{\Gamma}^{2}}{h}+\frac{g}{2} h^{2}\right]_{-L}^{+L}
$$

Denoting by $T$ the earliest time at which one of the two waves reaches the domain boundary, using the boundary conditions $h(-L, t)=h_{L}, h(+L, t)=h_{R}, q_{\Gamma}(-L, t)=q_{\Gamma}(+L, t)=0,0 \leq t \leq T$, bearing in mind that the storage porosity $\phi_{\Omega}$ is uniform over the domain, rearranging yields

$$
\partial_{t} \int_{-L}^{+L} q \mathrm{~d} x=\frac{\phi_{\Gamma}}{\phi_{\Omega}}\left(1-\mu_{x x}\right) \frac{g}{2}\left(h_{L}^{2}-h_{R}^{2}\right), \quad 0 \leq t \leq T
$$

where. Integrating the above equation between 0 and $t$ with the initial condition $q(x, 0)=0 \forall x$ leads to

$$
\int_{-L}^{+L} q \mathrm{~d} x=\frac{\phi_{\Gamma}}{\phi_{\Omega}}\left(1-\mu_{x x}\right) \frac{g}{2}\left(h_{L}^{2}-h_{R}^{2}\right) t, \quad 0 \leq t \leq T
$$


showing that the integral of the $x$-momentum increases linearly with time. In the absence of momentum dissipation, setting $\mu_{x x}=0$ leads to a standard momentum balance:

$$
\int_{-L}^{+L} q \mathrm{~d} x=\frac{\phi_{\Gamma}}{\phi_{\Omega}} \frac{g}{2}\left(h_{L}^{2}-h_{R}^{2}\right) t, 0 \leq t \leq T
$$

Figure 8 shows the variations of $\int_{-L}^{+L} q \mathrm{~d} x$ with time. The solid line represents the result of the flux balance on the right-hand side of equation (18), the dotted lines represent the values obtained from the numerical simulations. Note that all dotted lines do not cover the same time span because, depending on model geometry and IVP left and right states, $T$ is not identical for all simulations. Table 8 in Appendix A.3 gives the numerical values of the left- and right-hand side members of equation (18) and the ratio of the two for the 42 simulations.

In almost all cases, the integral of the $x$-momentum is observed to increase linearly with time. Two slight deviations from the linear behaviour can be observed, for simulations M4-R02 and M4-R03. This deviation cannot be attributed with absolute certainty to the development of the perturbations mentioned in Subsection 5.1, because it is not observed for Simulations M1b-R04 and M5-R04, where a perturbation is also observed to develop. The remaining 40 simulations show a remarkable agreement with a linear trend. When the connectivity porosity $\phi_{\Gamma}$ is isotropic (M1a-c, M2-M5), the amount of dissipation is almost independent of $\phi_{\Gamma}$. As a matter of fact, the dotted curves for M1b, M2-5 are very close to each other. The amount of dissipation larger than in the isotropic case when the porosity $\phi_{2}$ of the lateral streets is larger than the porosity $\phi_{1}$ of the longitudinal streets (Model M4). It is smaller than in the isotropic case when the porosity of the lateral streets is smaller than that of the longitudinal streets (Model M5). The amount of dissipation is influenced by the amplitude of the Riemann problem: the larger $h_{L}$, the larger the dissipation.

When the Riemann problem is not aligned with one of the main street directions, additional dissipation occurs. This is illustrated by Figure9. The momentum balance is carried out for Models M1b and M4 combined with IVPs R07-18. The solid line shows the flux balance on the right-hand side of equation (18), the dashed lines replicate the results of IVPs R01-06 shown on Figure 8 the dotted lines show the results for IVPs R07-18. Figure 9 shows that momentum dissipation is increased significantly when the Riemann problem does not propagate along the main street directions. Besides, the dotted lines clearly have a non-zero curvature. This does not necessarily mean that the transient momentum dissipation model (9) is invalid in this case. However, it is clearly overridden by stronger terms. Since the integral of the $x$-momentum is not a linear function of time, these additional dissipation terms cannot be expected to take the form $(9)$. 

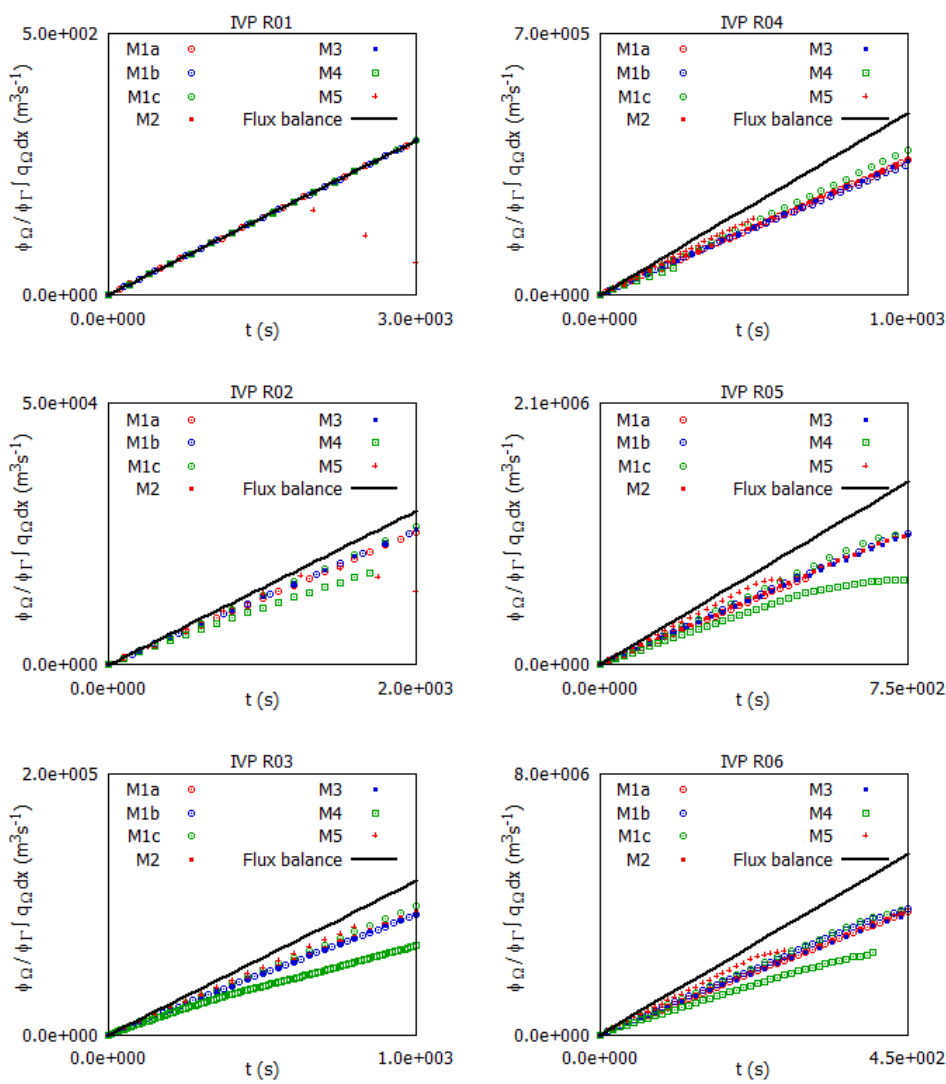

Figure 8: Transient momentum dissipation model. Total $x$-momentum as a function of time for Models M1-5 with IVPs R01-06. "Flux balance" is the solution obtained by setting $\mu_{x x}=0$. 

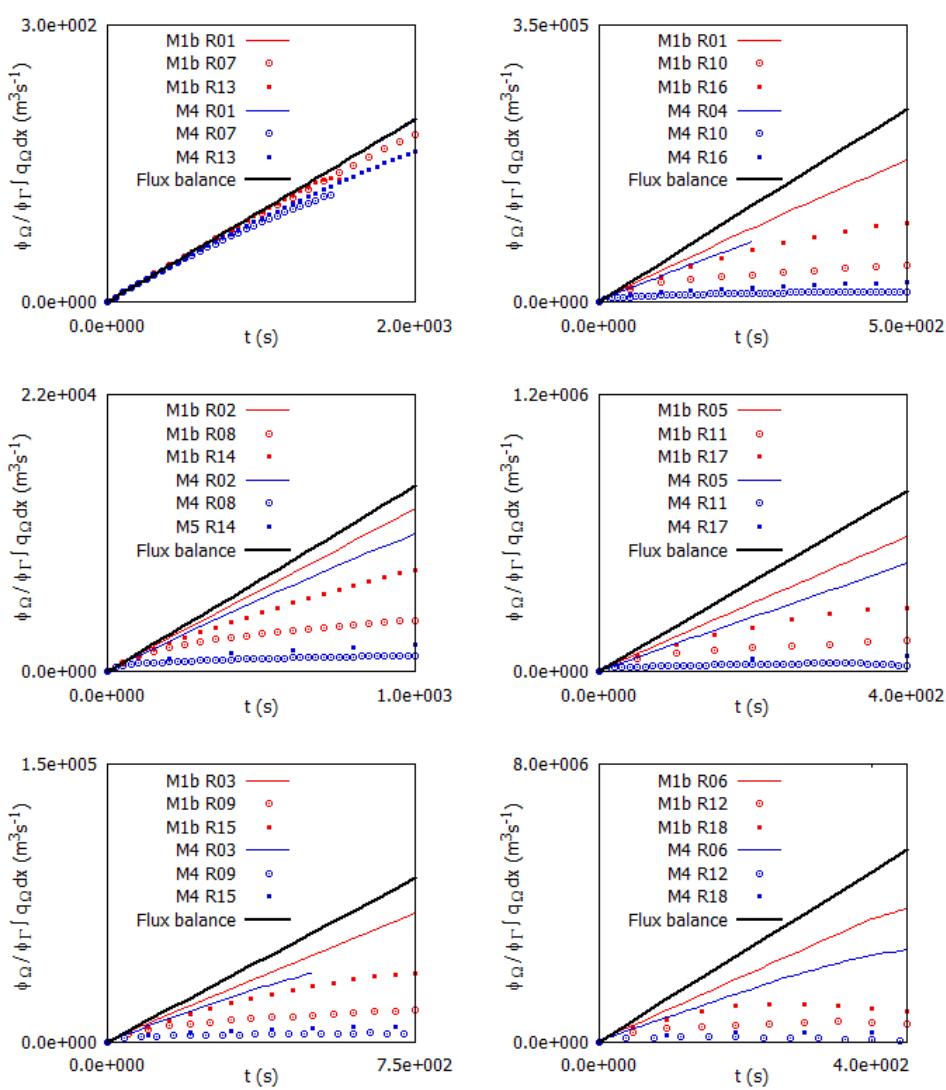

Figure 9: Momentum balance for Models M1b and M4, IVPs R07-R18.

\subsection{Building drag models}

The building drag models (equations (11, 12) ) are analysed only for square building layouts. For Models M1b, M2 and M3, BVPs B01-06 (see Section 3 and Figure 3 for details) are run until steady state is achieved. For each simulation, the building drag term is computed using the steady state solution. Square domains are defined by connecting the centroids of neighbouring building blocks (Figure 10). There are $N=12 \times 12=144$ such domains in the models. Each domain in the model is assigned a unique number $i=1, \ldots, N$. Integrating the steady state momentum balance over the $i$ th domain $\Omega_{i}$ gives

$$
\int_{\Omega_{i}} \mathbf{s}_{D} \mathrm{~d} \Omega=\int_{\Gamma_{i}} \mathbf{F}_{m} \mathbf{n} \mathrm{d} \Gamma
$$

where $\mathbf{n}$ is the normal unit vector to the boundary $\Gamma_{i}$ of $\Omega_{i}$. The domain-averaged building drag vector over the $i$ th domain is obtained from the above equation as

$$
\mathbf{s}_{D, i}=\frac{1}{\phi_{\Omega} L_{1} L_{2}} \int_{\Gamma_{i}} \mathbf{F}_{m} \mathbf{n} \mathrm{d} \Gamma
$$

The domain-averaged velocity vector is defined as:

$$
\mathbf{v}_{i}=\frac{\int_{\Omega_{i}} \mathbf{q d} \Omega}{\int_{\Omega_{i}} h \mathrm{~d} \Omega}
$$




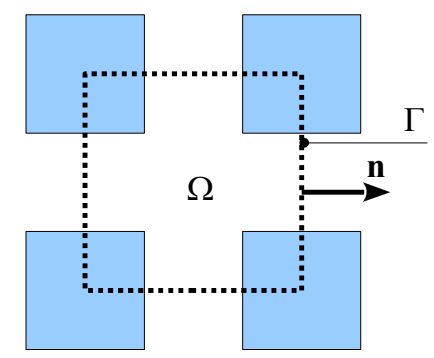

Figure 10: Control volume definition for building drag analysis. Definition sketch.

Likewise, it is possible to define a model-averaged velocity $\mathbf{v}_{M}$ and drag vector $\mathbf{s}_{D, M}$. Bearing in mind that all the rectangular domains in a given model have the same plan view area, one has

$$
\mathbf{v}_{M}=\frac{1}{N} \sum_{1}^{N} \mathbf{v}_{i}, \quad \mathbf{s}_{D, M}=\frac{1}{N} \sum_{1}^{N} \mathbf{s}_{D, i}
$$

The model-averaged vectors $(22)$ are used to calibrate the drag formulae $(11$ 12). For each of the six BVPs, $\mathbf{C}_{D}$ is calibrated by minimizing the distance

$$
D=\left|\mathbf{s}_{D, M}-\mathbf{s}_{D}\left(\mathbf{v}_{M}\right)\right|
$$

where $\mathbf{s}_{D}\left(\mathbf{v}_{M}\right)$ is the drag vector obtained by applying the drag formula (equation (11) or (12) to the domain-averaged velocity obtained from eq. (21). Note that the drag tensor is necessarily isotropic because the geometry itself is isotropic. Consequently, only one tensor coefficient needs to be calibrated. Once calibrated for a given BVP, the two building drag formulae are used to "predict" an average drag vector $\mathbf{s}_{D}\left(\mathbf{v}_{i}\right)$ over every rectangular domain $\Omega_{i}$ in the model. The validity of the model is checked by computing the RMSE between the "predicted" $\mathbf{s}_{D}\left(\mathbf{v}_{i}\right)$ vectors and the domain-averaged $\mathbf{s}_{D, i}$ vectors:

$$
E=\left[\frac{1}{N} \sum_{i=1}^{N}\left(\mathbf{s}_{D, i}-\mathbf{s}_{D}\left(\mathbf{v}_{i}\right)\right)^{2}\right]^{1 / 2}
$$

Table 5 shows the calibrated parameters and the validation error for the model [38] and the proposed model. The model (11) is extremely difficult to calibrate with the proposed approach. This could have been expected because calibrating this model involves the minimization of a single objective function with two degrees of freedom (the drag coefficient and the parameter $a$ ). The optimal parameter set is observed to be non-unique in the limit of algorithmic precision. Different optimization methods are seen to yield very different parameter sets. For this reason, Velickovic et al.'s model [38] was calibrated using the following, iterative procedure. In a first step, $a$ is fixed arbitrarily and the drag coefficient is calibrated using a genetic algorithm. In a second step, $a$ is changed manually so as to minimize the distance $D$. These two steps are repeated sequentially until no noticeable change is observed in the objective function. In contrast, calibrating the second drag model $(12)$ is straightforward. The parameter sets presented in Table 5 are calibrated with average errors $D \leq 10^{-15} \mathrm{~m}^{2} \mathrm{~s}^{-2}$, which is at least 10 orders of magnitude smaller than $\mathbf{s}_{D, M}$. The following conclusions are drawn from Table 5 .

Firstly, both models fail to provide an accurate description of the building drag. They both yield a posteriori errors $E$ larger than the norm of $\mathbf{s}_{D, M}$. With very similar $E$ values, none of the two models can be considered to behave significantly better than the other.

Secondly, the calibrated drag coefficients increase with the flow gradients (it is reminded that BVPs B01 to B06 involve increasing gradients between the upstream and downstream boundaries) and decrease with the porosity. The dependence on the flow gradient may indicate that the structure of the model is erroneous, thereby failing to account for certain effects. To give but one example, in [14] a drag coefficient proportional to the water depth was proposed because energy dissipation is assumed to occur with the same intensity all over the depth. The two tested models (11, 12) do not include such an assumption and failing to account for proportionality effects could be expected to 
yield large errors. Additional simulations, not reported here, show that making the drag proportional to the water depth does not contribute to reduce the variability of the drag coefficient and that $E$ is not reduced significantly. Other reasons must be sought to explain both models failing to predict the domain-averaged building drag.

\begin{tabular}{|c|c|c|c|c|c|c|c|c|}
\hline \multirow{2}{*}{ Model } & \multirow{2}{*}{ BVP } & \multirow{2}{*}{$\bar{h}(\mathrm{~m})$} & \multirow{2}{*}{$\left(\mathbf{s}_{D, M}\right)_{x}=\left(\mathbf{s}_{D, M}\right)_{y}$} & \multicolumn{3}{|c|}{ Velickovic et al. (2017) } & \multicolumn{2}{|c|}{ Present model } \\
\hline & & & & $C_{D, 11}=C_{D, 22}$ & $a$ & $E$ & $C_{D, 11}=C_{D, 22}$ & $E$ \\
\hline M1b & B01 & 1.00 & $-3.36 \times 10^{-5}$ & $1.58 \times 10^{-3}$ & 5.0 & $6.32 \times 10^{-5}$ & $1.36 \times 10^{-2}$ & $6.18 \times 10^{-5}$ \\
\hline M1b & В02 & 1.17 & $-2.73 \times 10^{-3}$ & $1.28 \times 10^{-3}$ & 5.0 & $5.47 \times 10^{-3}$ & $1.65 \times 10^{-2}$ & $5.38 \times 10^{-3}$ \\
\hline M1b & В03 & 2.38 & $-2.24 \times 10^{-2}$ & $3.08 \times 10^{-3}$ & 5.0 & $4.42 \times 10^{-2}$ & $3.78 \times 10^{-2}$ & $4.36 \times 10^{-2}$ \\
\hline M1b & B04 & 4.63 & $-9.41 \times 10^{-2}$ & $5.96 \times 10^{-3}$ & 5.0 & $1.84 \times 10^{-1}$ & $7.04 \times 10^{-2}$ & $1.81 \times 10^{-1}$ \\
\hline M1b & B05 & 9.25 & $-3.88 \times 10^{-1}$ & $1.19 \times 10^{-2}$ & 5.0 & $7.70 \times 10^{-1}$ & $1.38 \times 10^{-1}$ & $7.60 \times 10^{-1}$ \\
\hline M1b & B06 & 23.1 & -2.46 & $2.96 \times 10^{-2}$ & 5.0 & 4.86 & $3.44 \times 10^{-1}$ & 4.80 \\
\hline M2 & B01 & 1.00 & $-3.80 \times 10^{-5}$ & $2.91 \times 10^{-3}$ & 5.0 & $6.65 \times 10^{-5}$ & $2.44 \times 10^{-2}$ & $6.37 \times 10^{-5}$ \\
\hline M2 & В02 & 1.23 & $-4.21 \times 10^{-3}$ & $2.66 \times 10^{-3}$ & 5.0 & $8.19 \times 10^{-3}$ & $3.64 \times 10^{-2}$ & $7.90 \times 10^{-3}$ \\
\hline M2 & B03 & 2.31 & $-3.05 \times 10^{-2}$ & $4.52 \times 10^{-3}$ & 5.0 & $5.89 \times 10^{-2}$ & $6.29 \times 10^{-2}$ & $5.60 \times 10^{-2}$ \\
\hline M2 & B04 & 4.48 & $-1.29 \times 10^{-1}$ & $8.91 \times 10^{-3}$ & 5.0 & $2.48 \times 10^{-1}$ & $1.18 \times 10^{-1}$ & $2.35 \times 10^{-1}$ \\
\hline M2 & B05 & 9.00 & $-5.47 \times 10^{-1}$ & $1.83 \times 10^{-2}$ & 5.0 & $9.65 \times 10^{-1}$ & $2.42 \times 10^{-1}$ & $8.86 \times 10^{-1}$ \\
\hline M2 & B06 & 23.4 & & $4.78 \times 10^{-2}$ & 5.0 & 7.09 & $6.18 \times 10^{-1}$ & 6.60 \\
\hline M3 & B01 & 1.00 & $-2.05 \times 10^{-4}$ & $1.33 \times 10^{-3}$ & 5.0 & $6.10 \times 10^{-4}$ & $3.66 \times 10^{-2}$ & $7.17 \times 10^{-4}$ \\
\hline M3 & В02 & 1.17 & $-3.71 \times 10^{-3}$ & $2.25 \times 10^{-3}$ & 5.0 & $7.08 \times 10^{-3}$ & $3.11 \times 10^{-2}$ & $6.62 \times 10^{-3}$ \\
\hline M3 & Воз & 2.34 & $-3.01 \times 10^{-2}$ & $5.04 \times 10^{-3}$ & 5.0 & $5.73 \times 10^{-2}$ & $6.55 \times 10^{-2}$ & $5.38 \times 10^{-2}$ \\
\hline M3 & B04 & 4.59 & $-1.27 \times 10^{-1}$ & $1.00 \times 10^{-2}$ & 5.0 & $2.40 \times 10^{-1}$ & $1.25 \times 10^{-1}$ & $2.25 \times 10^{-1}$ \\
\hline M3 & B05 & 9.35 & $-5.30 \times 10^{-1}$ & $2.06 \times 10^{-2}$ & 5.0 & 1.01 & $2.54 \times 10^{-1}$ & $9.51 \times 10^{-1}$ \\
\hline M3 & B06 & 23.9 & -3.46 & $5.36 \times 10^{-2}$ & 5.0 & $6.63 \times 10^{-4}$ & 0.66 & 6.26 \\
\hline
\end{tabular}

Table 5: Building drag model analysis. $\left(\mathbf{s}_{D, M}\right)_{x}$ and $\left(\mathbf{s}_{D, M}\right)_{y}$ are respectively the $x-$ and $y$-components of $\mathbf{s}_{D, M}, C_{D}$ and $a$ are calibrated values.

Figure 11 illustrates the simulation results for Model M1b and BVP B03. The results for the remaining BVPs are plotted in Appendix A.4 for the sake of paper readbility (see Figures 24 28). . The behaviour of Models M2 and M3 is essentially similar and is not plotted for the sake of paper conciseness. The upper row in Figures 11, 24, 28 shows the $N$ domain-averages $\mathbf{v}_{i}$ and $\mathbf{s}_{D, i}$ computed using equations 20, 21). The lower row shows the predicted drag vectors $\mathbf{s}_{D}\left(\mathbf{v}_{i}\right)$ computed using the drag models 11 12.

Examining the flow velocity field $\mathbf{v}_{i}$ (upper left scatter plots) confirms the analysis of the flux closure models in Section 4. In most simulations, the flow velocity field is strongly aligned with the main two street directions. Only a small number of domain averages are scattered along the diagonal. The alignment is all the more pronounced as the flow gradients and velocities are smaller. Only for BVPs B05 and B06 is it possible to observe a deviation from the main directions. With upstream water depths of $20 \mathrm{~m}$ and $50 \mathrm{~m}$ respectively, these two BVPs, however, represent extreme flow configurations that are unlikely to occur in real-world situations.

The statistical distribution of the drag vector $\mathbf{s}_{D, i}$, in contrast, indicates a strongly polarized field in the diagonal direction. The anisotropy of the drag field increases with the flow intensity. Comparing the drag vector distributions to the flow velocity distributions does not allow any clear mapping from $\mathbf{v}_{i}$ to $\mathrm{s}_{D, i}$ to be identified. Another salient feature of the drag field is the spatial distribution of the drag values. While the velocity field is characterized by a large number of domains with extreme velocities, the drag field counts a large number of small values (compared to the average) and a few extreme values.

The scatter plots for the building drags $\mathbf{s}_{D}\left(\mathbf{v}_{i}\right)$ predicted by the two models 11, 12 are shown on the lower row on Figures 11] 24] 28. The statistical distribution for the modelled drags are extremely different from those of the domain average $\mathbf{s}_{D, i}$. The structure of the predicted drag field obviously follows that of the velocity field. With Velickovic et al.'s model [38], the predicted drag field remains preferentially aligned along the main street directions. With the proposed model $(12)$, the 
predicted drag field exhibits a weaker alignment with the main street directions. However, in both cases, the predicted drag vector scatter plots are very different from those computed from the fine grid simulations.
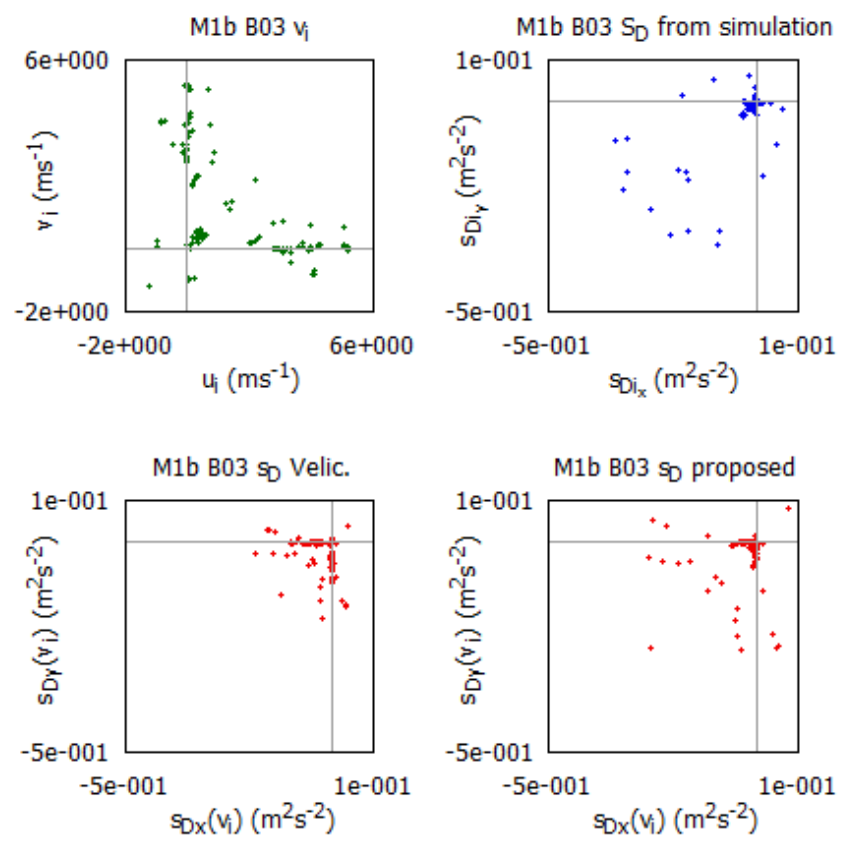

Figure 11: Simulation M1b-B03. Top: domain-averaged velocity vectors $\mathbf{v}_{i}$ (left) and drag vectors $\mathbf{s}_{D, i}$ (right). Bottom: predicted $\mathbf{s}_{D}\left(\mathbf{v}_{i}\right)$ using Velickovic et al.'s model (left) and the proposed formula (right).

\section{Summary of results}

Since the objective is to assess the accuracy of closure models for interfaces, the results are interpreted in the local coordinate system attached to a given interface. To this end, the normal and tangent unit discharges to $\Gamma$ are defined respectively as follows

$$
q_{n}=\mathbf{q}_{\Gamma} \cdot \mathbf{n}, q_{t}=\mathbf{q}_{\Gamma} \cdot \mathbf{t}
$$

where $\mathbf{n}$ and $\mathbf{t}$ are respectively the normal and tangent unit vectors to $\Gamma$. Two types of interface $\Gamma$ are used in the present study: $x$-facing interfaces, with $\mathbf{n}=(1,0)^{T}$, and $y$-facing interfaces, with $\mathbf{n}=(0,1)^{T}$. Table 6 gives the expressions for the various elements of the flux tensor in the local coordinate system attached to the interface for these two interface orientations.

\begin{tabular}{cccccc}
\hline Interface facing & $F_{11}=q_{n}$ & $F_{12}=q_{t}$ & $F_{21}=\frac{q_{n}^{2}}{h}+\frac{g}{2} h^{2}$ & $F_{22}=F_{31}=\frac{q_{n} q_{t}}{h}$ & $F_{32}=\frac{q_{t}^{2}}{h}+\frac{g}{2} h^{2}$ \\
\hline$x$ & $q$ & $r$ & $\frac{q^{2}}{h}+\frac{g}{2} h^{2}$ & $\frac{q r}{h}$ & $\frac{r^{2}}{h}+\frac{g}{2} h^{2}$ \\
$y$ & $r$ & $-q$ & $\frac{r^{2}}{h}+\frac{g}{2} h^{2}$ & $-\frac{q r}{h}$ & $\frac{q^{2}}{h}+\frac{g}{2} h^{2}$ \\
\hline
\end{tabular}

Table 6: Components of the flux tensor in the local coordinate system attached to the interface.

Table 7 summarizes the results obtained in Sections 4-5 for the various flux closures and momentum source term models. For the reader's convenience, the relevant subsections, figures and tables are also mentioned in Table 7. The main results are the following. 
- The closure model for the water depth is correct and the interface pressure term is computed correctly in all simulations for the SP, IP and DIP models.

- The DIP model is the only model that yields a correct estimate for the normal mass flux $q_{n}$. The SP and IP yield underestimated normal discharges compared to the reference data set.

- All three models fail to reproduce the preferential directions for the interface flux $\mathbf{q}_{\Gamma}$ when the flow is not aligned with the main directions of the street networks and/or if the street network is not orthogonal. This results in wrong estimates for the momentum fluxes $q_{n}^{2} / h$ and $q_{n} q_{t} / h$. The DIP model, however, yields a smaller error than the SP and IP models.

- The transient momentum dissipation model active for positive waves is validated.

- None of the building drag models proposed so far in the literature (including the model proposed in the present paper) succeeds to reproduce the reference drag term fields.

\begin{tabular}{|c|c|c|}
\hline Element & $\begin{array}{l}\text { Section of } \\
\text { paper }\end{array}$ & Result(s) \\
\hline $\begin{array}{l}\text { Normal mass flux } \\
F_{11}\end{array}$ & $\begin{array}{l}4.2, \text { Figs. } 12, \\
14-17\end{array}$ & SP and IP closures inaccurate. DIP closure accurate. \\
\hline $\begin{array}{l}\text { Transverse mass } \\
\text { flux } F_{12}\end{array}$ & $\begin{array}{l}4.2, \text { Figs. } 13, \\
14-17\end{array}$ & $\begin{array}{l}\text { Closure accurate for all models when the street network is orthogonal and } \\
\text { the flow is aligned with one of the street axes. In other situations, all models } \\
\text { fail to account for the alignment of } \mathbf{q}_{\Gamma} \text { with the street axes. }\end{array}$ \\
\hline $\begin{array}{l}\text { Hydrostatic } \\
\text { pressure term } \frac{g}{2} h^{2} \\
\text { in } F_{21} \text { and } F_{32}\end{array}$ & 4.3, Fig. 6 & Closure accurate for all models \\
\hline $\begin{array}{l}\text { Momentum flux } \\
\frac{q_{n}^{2}}{h} \text { in } F_{21} \text { and } F_{32}\end{array}$ & $\begin{array}{l}4.3, \text { Figs. } 18, \\
21,22\end{array}$ & $\begin{array}{l}\text { SP and IP closures inaccurate. DIP closure accurate when the street } \\
\text { network is orthogonal and the flow is aligned with one of the street axes. In } \\
\text { other situations, the flux is underestimated substantially. Correction factor } \\
\chi \geq 1 \text { needeed. }\end{array}$ \\
\hline $\begin{array}{l}\text { Momentum flux } \\
F_{22}=F_{31}=\frac{q_{n} q_{t}}{h}\end{array}$ & 4.3, Fig. 19 & $\begin{array}{l}\text { All closures strongly inaccurate when the street network is not orthogonal } \\
\text { and/or the flow is not aligned with one of the main street axes }\end{array}$ \\
\hline $\begin{array}{l}\text { Momentum flux } \\
\frac{q_{t}^{2}}{h} \text { in } F_{32}\end{array}$ & 4.3, Fig. 20 & $\begin{array}{l}\text { All closures inaccurate. Of secondary importance because this term is not } \\
\text { involved in the momentum balance. }\end{array}$ \\
\hline $\begin{array}{l}\text { Momentum } \\
\text { dissipation term } \\
\mathbf{s}_{T}\end{array}$ & $\begin{array}{l}5.1-2, \text { Figs. } 8, \\
9, \text { Table } 8\end{array}$ & $\begin{array}{l}\text { Model accurate. Dissipation coefficient more sensitive to street width ratio } \\
\text { than to porosity. }\end{array}$ \\
\hline $\begin{array}{l}\text { Building drag term } \\
\mathbf{s}_{D}\end{array}$ & $\begin{array}{l}5.3, \text { Figs. } 11, \\
24-28, \text { Table } 5 .\end{array}$ & Both models inaccurate \\
\hline
\end{tabular}

Table 7: Summary of the analysis results for the flux and source term models.

\section{Conclusions}

A base of 96 refined shallow water simulations over periodic street networks is set up. 78 IVPs and 18 BVPs combined with 9 different geometries are analysed to assess the governing assumptions of the SP, IP and DIP models. The purpose is to answer Questions Q1-Q4 in the Introduction section. Validation is carried out by comparing the closure relationships of the $\mathrm{SP} / \mathrm{IP} / \mathrm{DIP}$ models to the refined simulation 
results on the scale of a building block. This contrasts with more usual, indirect validation techniques, that use global indicators operating on the flow solution over the complete domain. Questions Q1-4 are answered as follows.

A1. The DIP model is the more accurate of the three models. It yields accurate estimates for the normal mass flux and the pressure force, while the SP and IP do not. The normal momentum flux is correctly computed by the DIP closure provided that the street network is orthogonal and the flow propagates along one of the principal directions of the streets. Other situations generate erroneous normal and transverse momentum fluxes. The reason for this is found in the closures failing to account for the preferential alignment of the flow along the axes of the streets, a feature already observed in [14] for the Sacramento application. The transient momentum dissipation model introduced in [14] is also validated. The latest building drag formula proposed so far in the literature [38] is also invalidated, as well as a modified formula. Testing previously proposed building drag models [14, 34] would have been meaningless because these models fail to fulfil basic requirements of the building drag.

A2. The connectivity porosity is obviously not sufficient to an accurate computation of the fluxes and source terms. Simulations involving oblique waves the street network show that none of the SP, IP and DIP models accounts correctly for the alignment of the flow velocity field with the main street axes. Consequently, the connectivity porosity should be supplemented with another indicator giving the direction of the flow. As far as the transient dissipation term is concerned, simulations involving identical porosities in both directions shows that the dissipation rate is also a function of the street width ratio.

A3. As far as possible improvements of the DIP model are concerned, four main paths may be considered.

The non-uniform velocity field across the street section mandates the introduction of a momentum distribution coefficient $\chi \geq 1$ in the momentum fluxes. A model is still to be proposed for the law governing $\chi$ as a function of the angle between the velocity vector and the street axes. As far as real-world applications of porosity models are concerned, introducing a model for $\chi$ would have two positive consequences. First, the wave propagation properties of the porosity model would be improved. Second, $\chi$ is a measure of the variabiliity of the flow field at the subgrid scale. It may thus bring added value in assessing the distribution of the the risk (that is a function of both the water depth and the flow velocity) on the subgrid scale.

A law allowing for the preferential alignment of the velocity vectors with the street axes is needed. Together with the momentum distribution coefficient, this would allow for a correct representation of the flow velocity fields and wave propagation properties along street networks. Such a closure should be free from the consistency issues identified in [12].

A model (empirical or theoretical) should also be proposed for the coefficients $\mu_{i j}$ of the momentum dissipation tensor as a function of the street width ratios and flow variables. In the current state of development of the DIP model, the coefficients $\mu_{i j}$ must be calibrated. While this increases the flexibility of the model, adding parameters puts a heavier burden on the modeller, because their calibration requires additional runs and increases the needs for data collection. In the field of urban floods, however, data collection is not an easy task, especially in the case of the DIP momentum dissipation model, that is active only under transient conditions.

Lastly, the building drag source term also requires improvements. None of the source terms explored so far in the literature of shallow water porosity models gives satisfactory results after calibration, a likely indication that the 
structure of the model should be revised. The possibility of adapting flow distribution models based on two-dimensional mass and momentum balance considerations such as [25] should be considered. Improving the building drag model will yield improved flow velocity and discharge distributions within the streets, especially when the flow pattern is strongly two-dimensional (locally converging or diverging flows, as explored in Section 5).

A4. The following recommendations can be made for the design of experiments for the validation of the flux and source term closures in shallow water porosity models.

Experimental validation should not focus only on the water depth field, but also on the flow velocity (or unit discharge) fields. As shown by the present experiments, the SP, IP and DIP models yield identically accurate water depth closures. Only comparing the unit discharge/flow velocity closures to the refined flow simulations allows the three models to be discriminated. The numerical experiments reported here also indicate that the flow velocity profiles cannot be assumed uniform across the streets in a number of situations. Consequently, measuring only discharges within the streets does not suffice to achieve an accurate momentum balance. Exploring the detailed velocity profiles across the streets is mandatory.

Secondly, all three models identically fail to reproduce the alignment of the flow along the street axes when the average flow is oblique to the street directions. Consequently, recording the longitudinal components of the flow velocity does not suffice and a genuine two-dimensional flow field exploration is needed.

Steady state experiments must be complemented with transient experiments. To start with, only transient experiments will allow the existence of transient momentum source terms to be confirmed experimentally. However, systematic urban dambreak problems with a detailed investigation of the water depth and flow fields are extremely time-consuming to realise experimentally. This is due to the long settling times needed to achieve well-controlled, still water initial conditions and the need to perform several replicates of the same experiment. Simpler wave propagation experiments could be used to validate the longitudinal mass and momentum flux closures. A possible setup is the following: starting from a steady initial state with water flowing along the main street direction, measuring the propagation speeds of small perturbations in the upstream and downstream directions across several block periods would yield the eigenvalues of the wave propagation problem. Erroneous wave propagation speeds would indicate erroneous flux models. Such experiments would take only a few seconds and could be replicated a large number of times, thus allowing a wide range of flow configurations to be explored.

It is acknowledged that the simulations reported here are highly theoretical. The street networks are strictly rectilinear and periodic. All buildings are aligned exactly, the bottom is flat and frictionless. Non-ideal situations are likely to yield increased source terms in the momentum conservation equation. Some of the conclusions drawn in the present study may may lose strength when additional topographic and frictional effects are included, and this issue should also be addressed in future experimental

validation. Nevertheless, porosity models performing correctly against ideal situations is seen as an indispensable prerequisite to their application to real-world situations involving complex geometries.

\section{Appendix - Additional figures and tables}

\section{A.1 Figures for Section 4.2}

The figures hereafter support the analysis of the mass flux closure model presented in Subsection 4.2. 

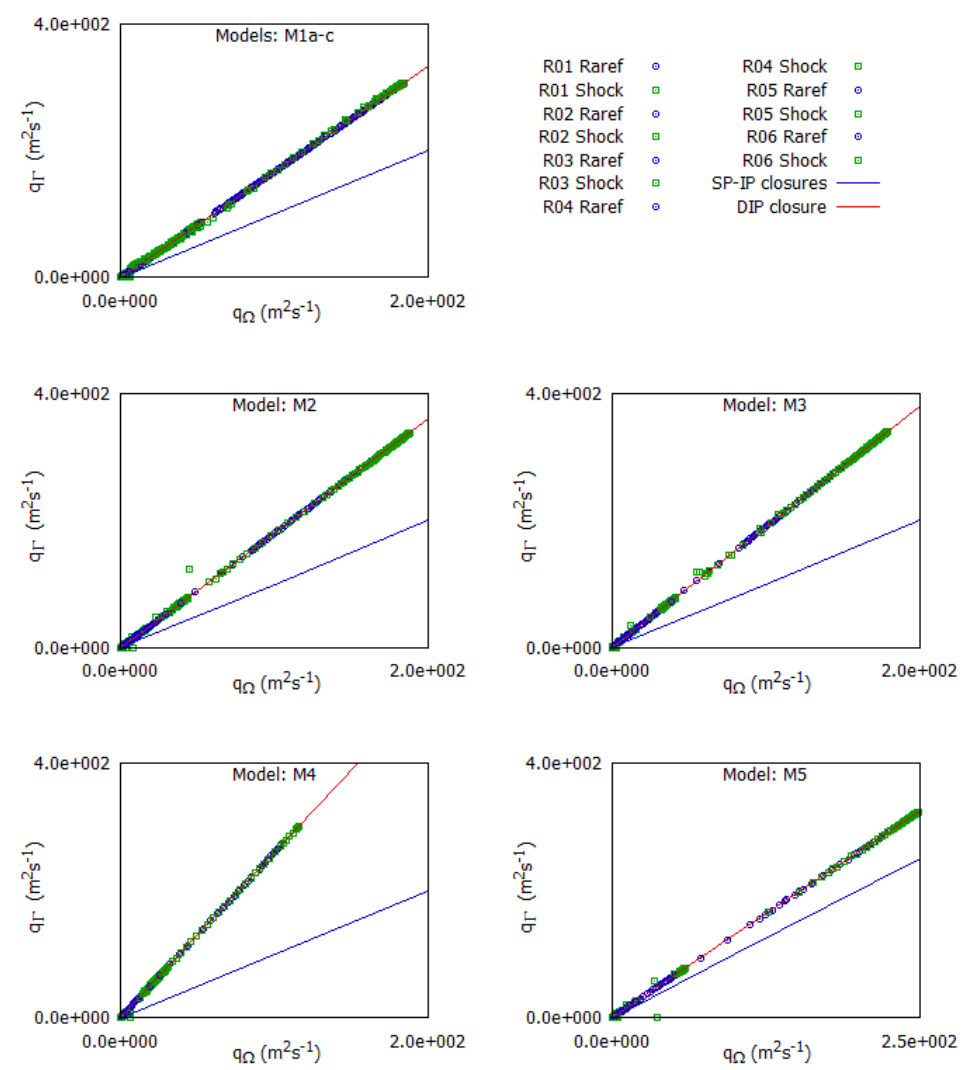

Figure 12: Models M1-M5, IVPs R01-06. Scatter plot for $\left(q_{\Omega}, q_{\Gamma}\right)$ over $x$-facing interfaces. 

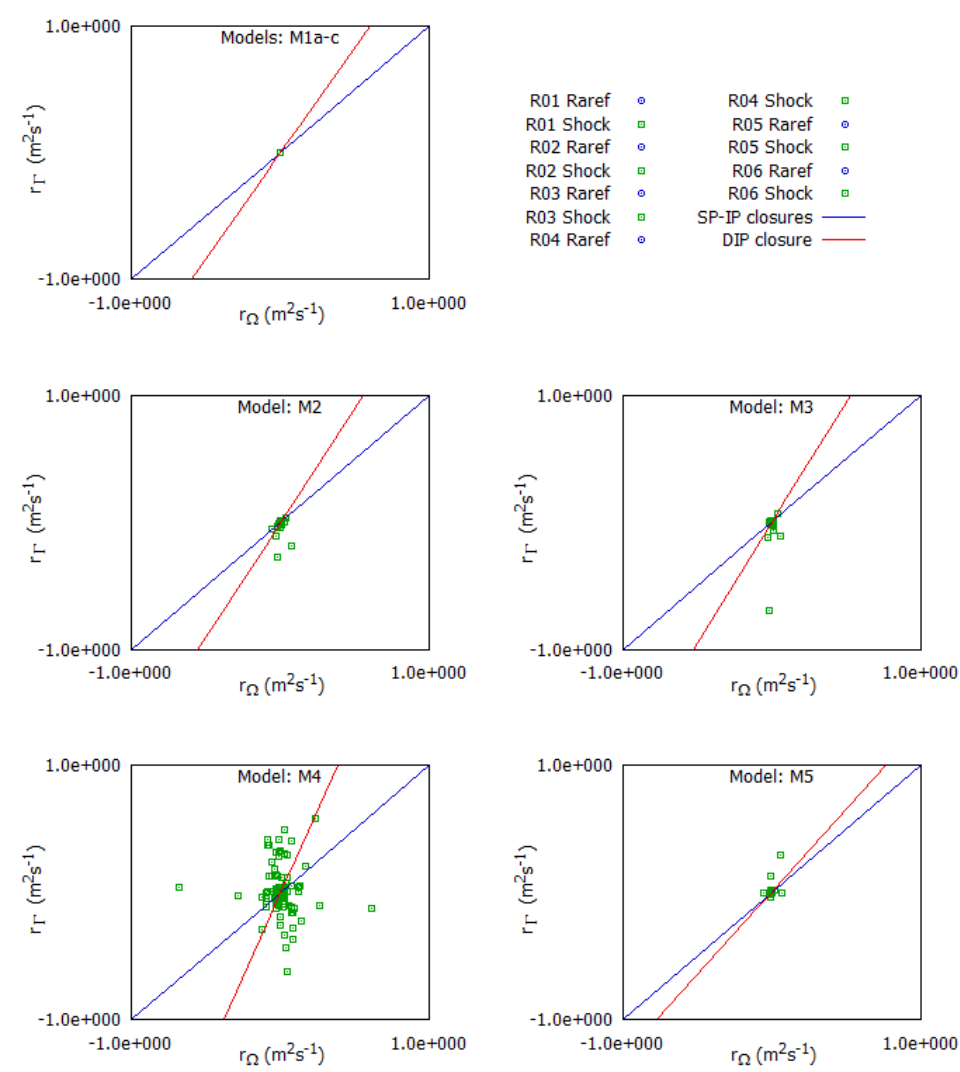

Figure 13: Models M1-M5, IVPs R01-06. Scatter plot for $\left(r_{\Omega}, r_{\Gamma}\right)$ over $x$-facing interfaces.
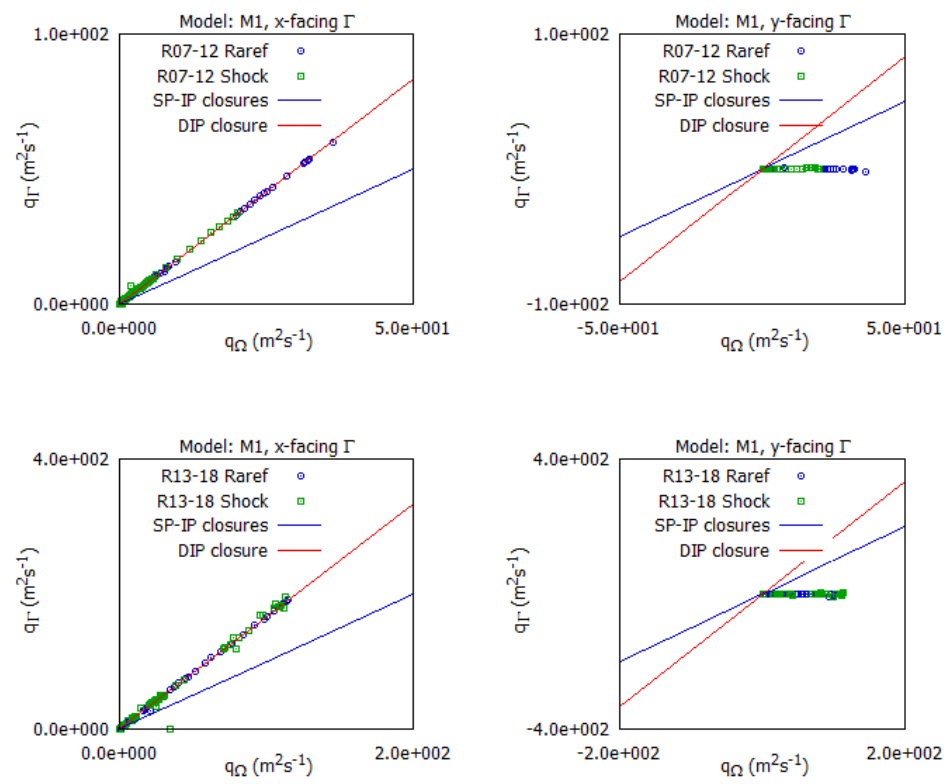

Figure 14: Model M1b, IVPs R07-18. Scatter plot for $\left(q_{\Omega}, q_{\Gamma}\right)$ over $x$ - and $y$-facing interfaces. 

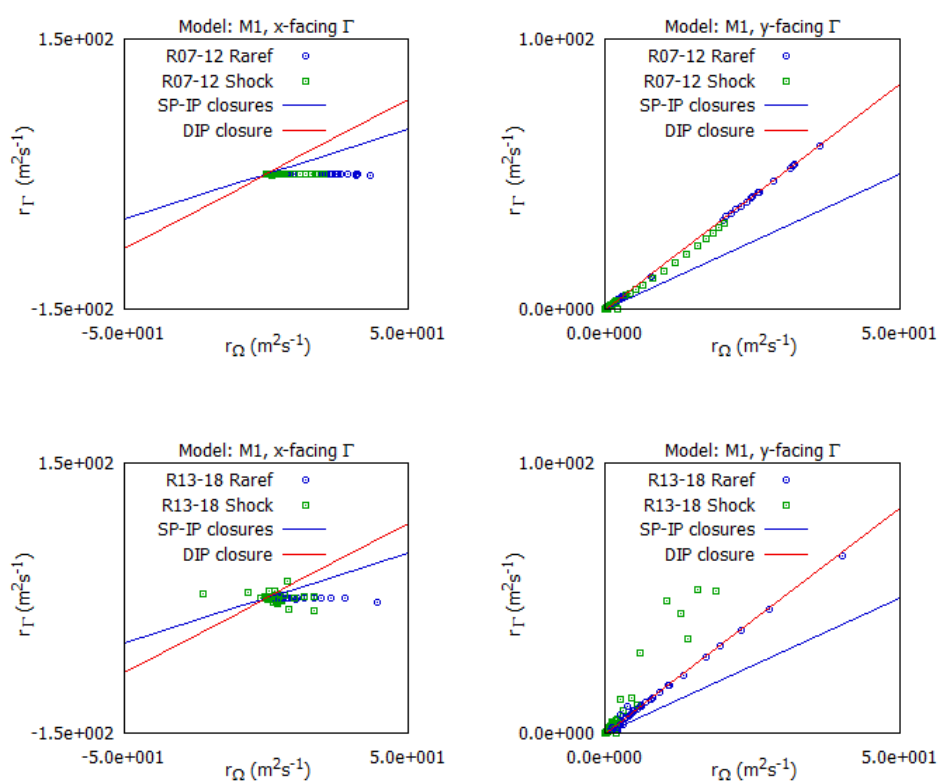

Figure 15: Model M1b, IVPs R07-18. Scatter plot for $\left(r_{\Omega}, r_{\Gamma}\right)$ over $x-$ and $y$-facing interfaces.
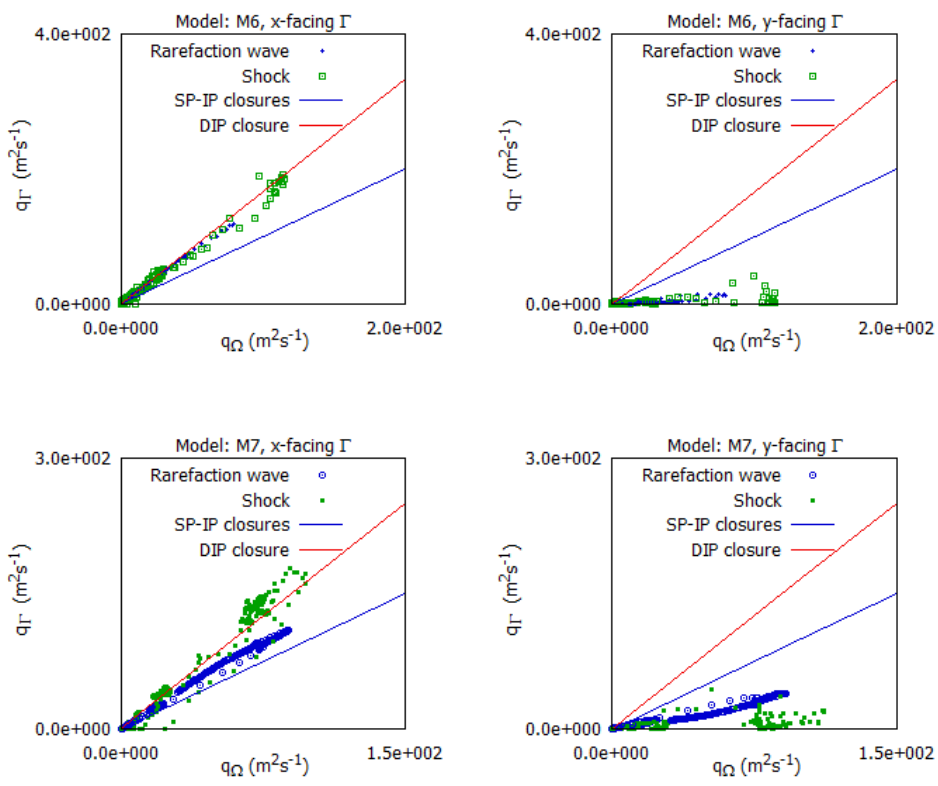

Figure 16: Models M6-7, IVPs R01-06. Scatter plot for $\left(q_{\Omega}, q_{\Gamma}\right)$ over $x$ - and $y$-facing interfaces. 

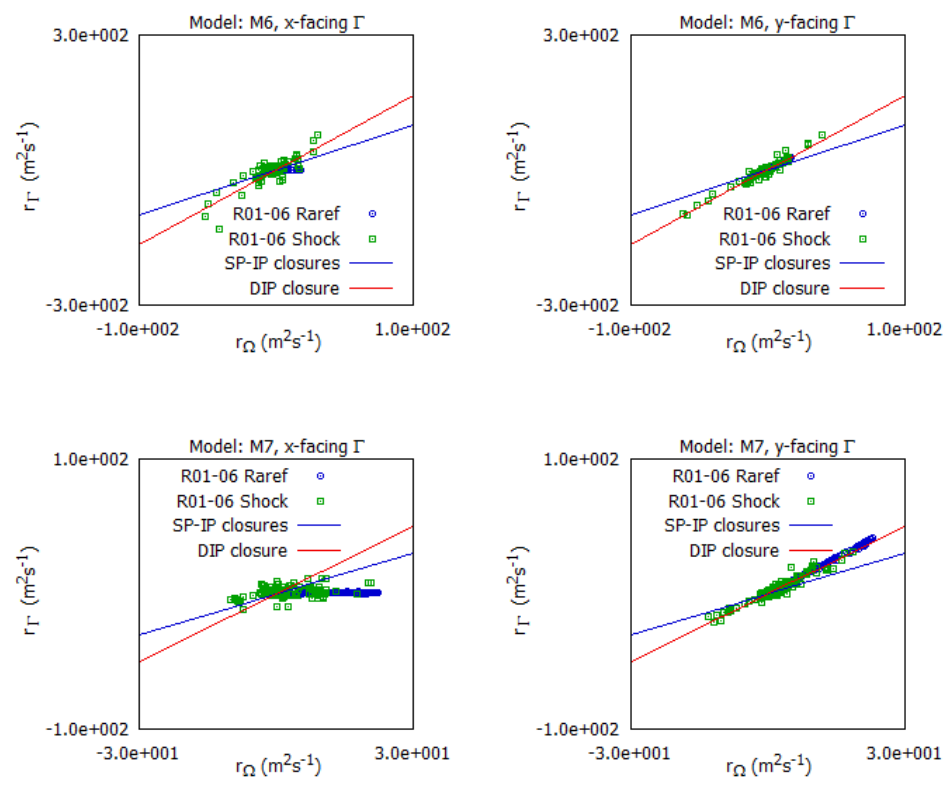

Figure 17: Models M6-7, IVPs R01-06. Scatter plot for $\left(r_{\Omega}, r_{\Gamma}\right)$ over $x-$ and $y$-facing interfaces.

\section{A.2 Figures for Subsection 4.3}

The figures hereafter support the analysis of the mass flux closure model presented in Subsection 4.2. 


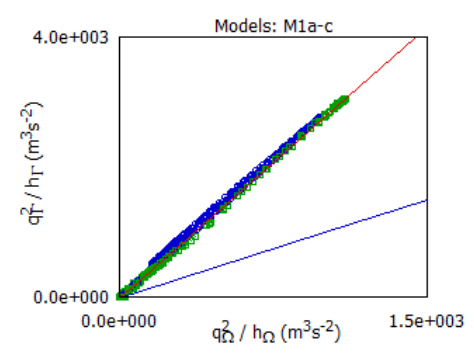

R01-06 Raref o

R01-06 Shock

SP-IP closures
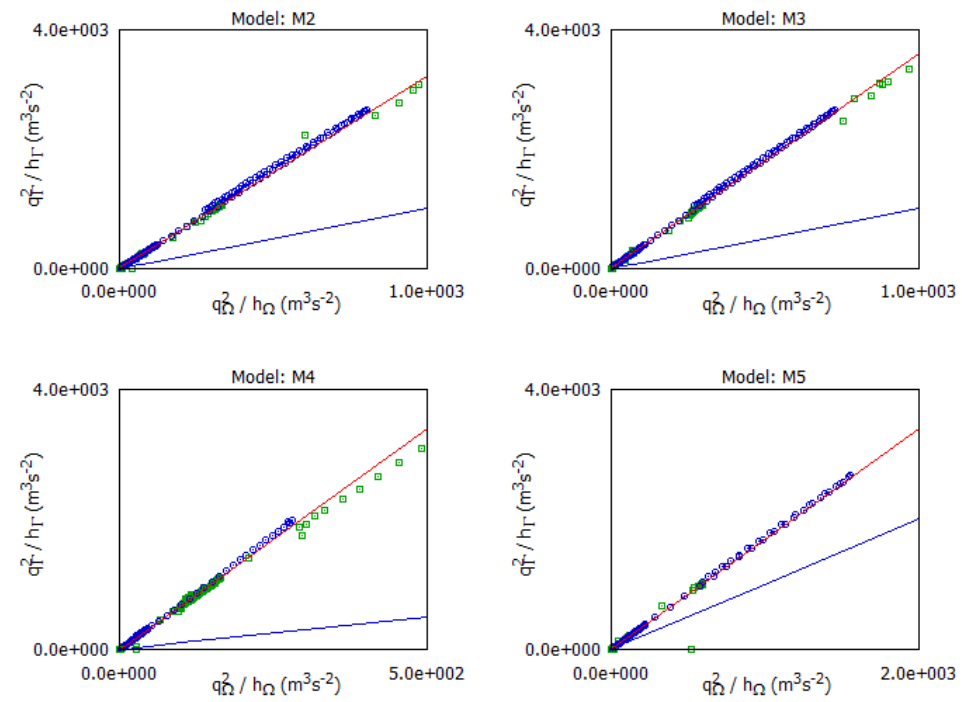

Figure 18: Models M1-5, IVPs R01-06. Scatter plot for the $\frac{q^{2}}{h}$ momentum discharge closure over $x$-facing interfaces. 

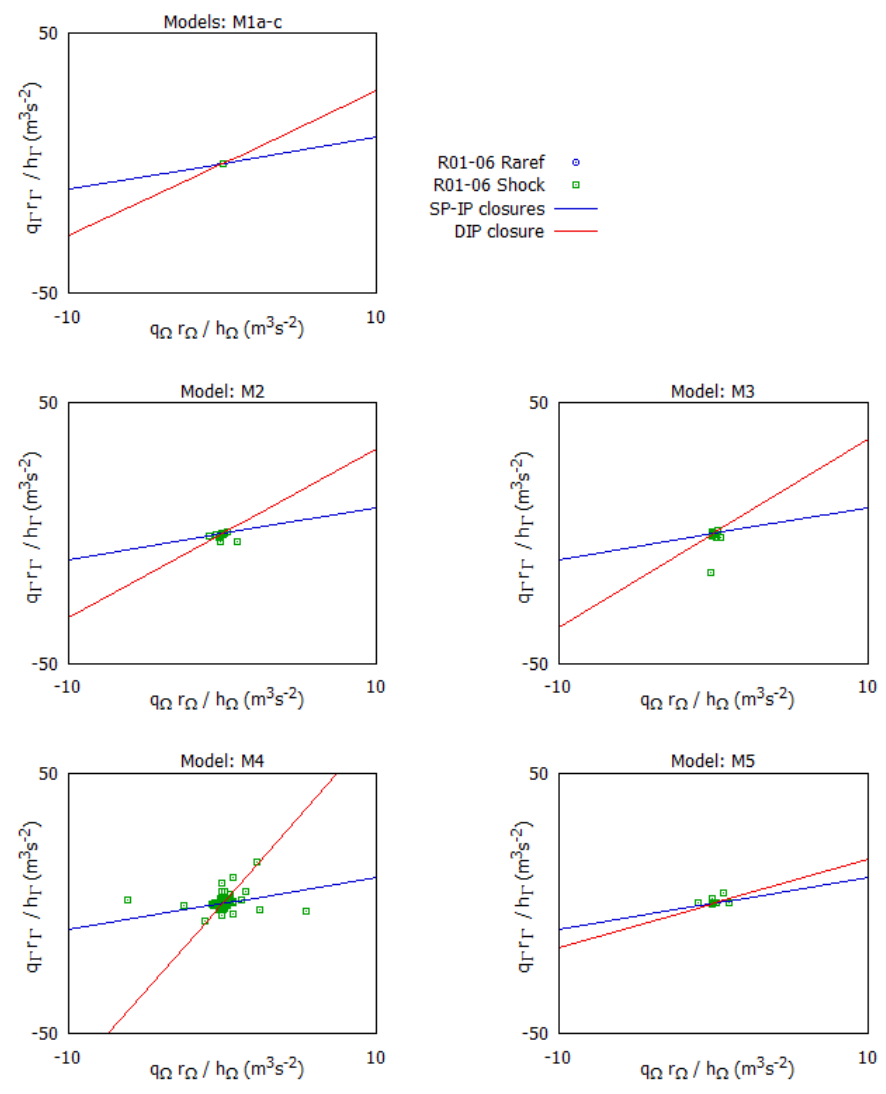

Figure 19: Models M1-5, IVPs R01-06. Scatter plot for the $\frac{q r}{h}$ momentum discharge closure over $x$-facing interfaces. 

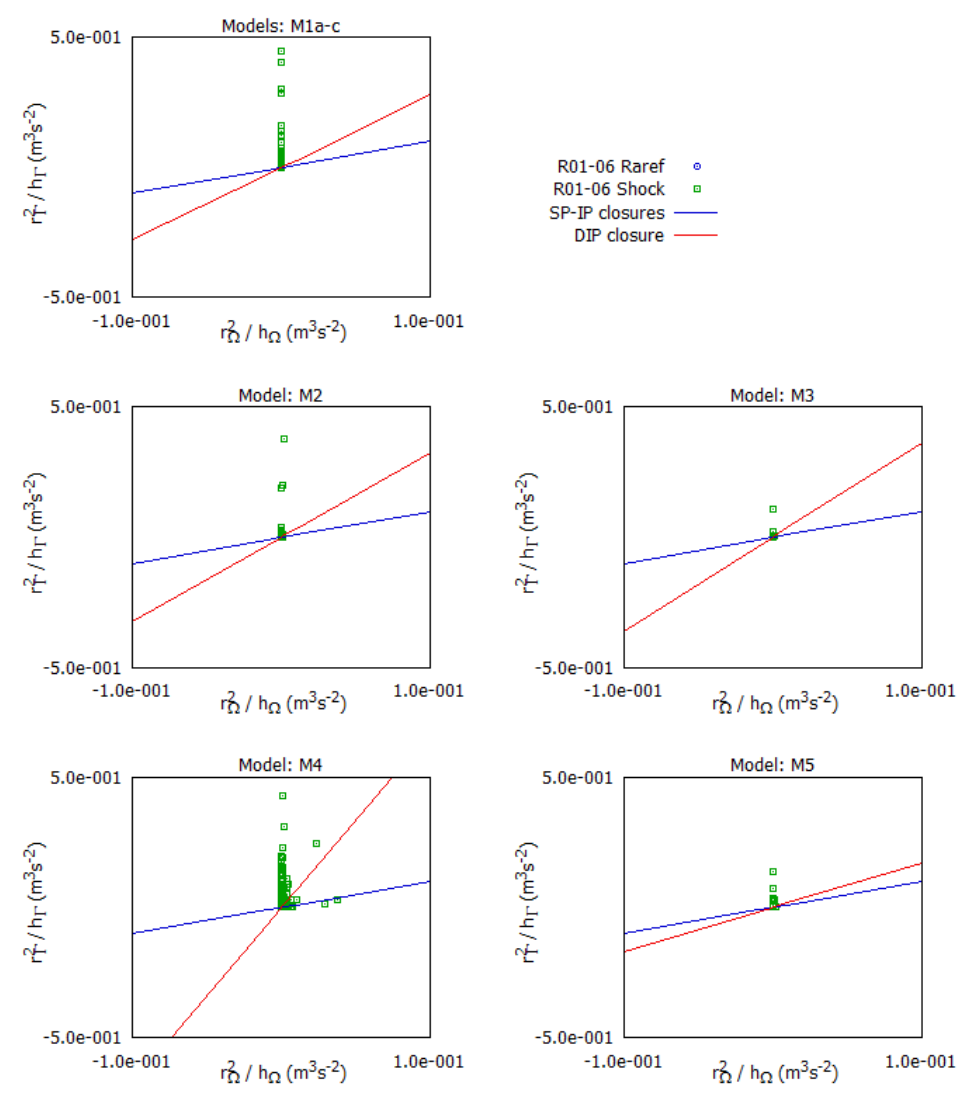

Figure 20: Models M1-5, IVPs R01-06. Scatter plot for the $\frac{r^{2}}{h}$ momentum discharge closure over $x$-facing interfaces. 

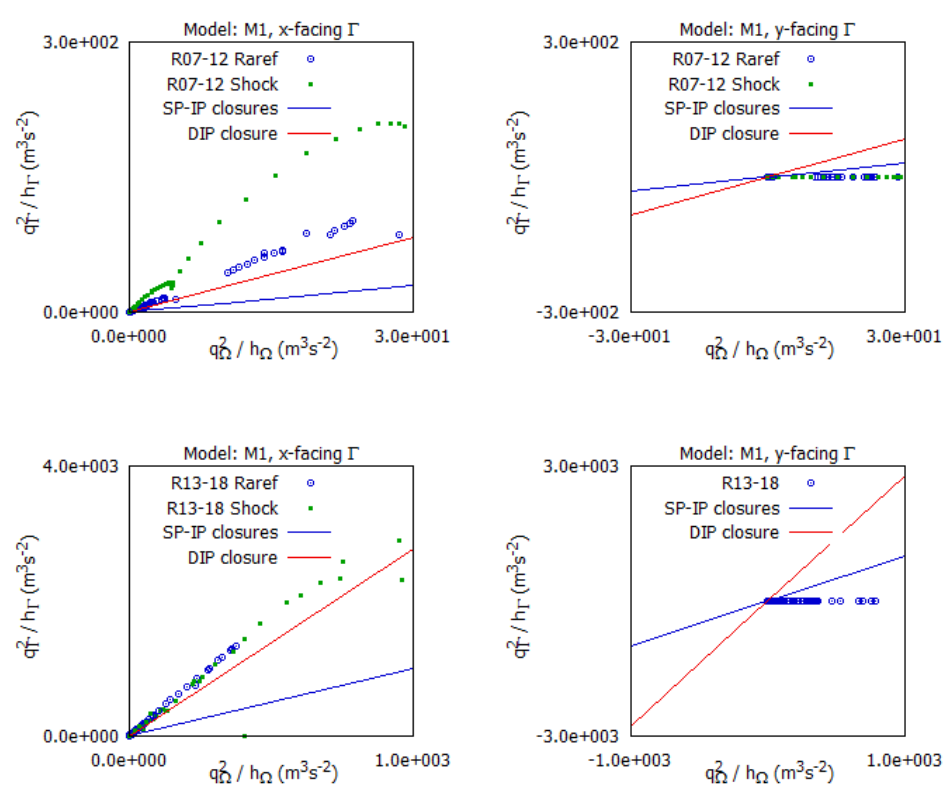

Figure 21: Model M1b, IVPs R07-18. Scatter plot for the $\frac{q^{2}}{h}$ momentum discharge closure over $x-$ and $y$-facing interfaces.
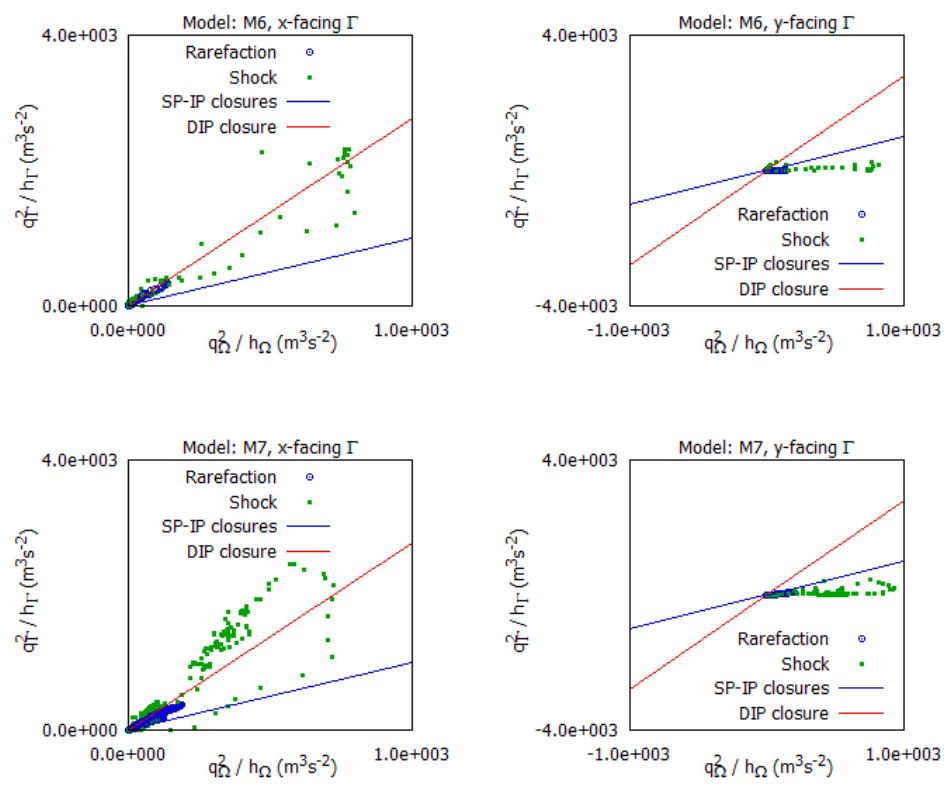

Figure 22: Models M6 and M7, IVPs R01-07. Scatter plot for the $\frac{q^{2}}{h}$ momentum discharge closure over $x-$ and $y$-facing interfaces.

\section{A.3 Transient momentum dissipation analysis}

Figure and Table 5 support the analysis presented in Subsections 5.1-2. 


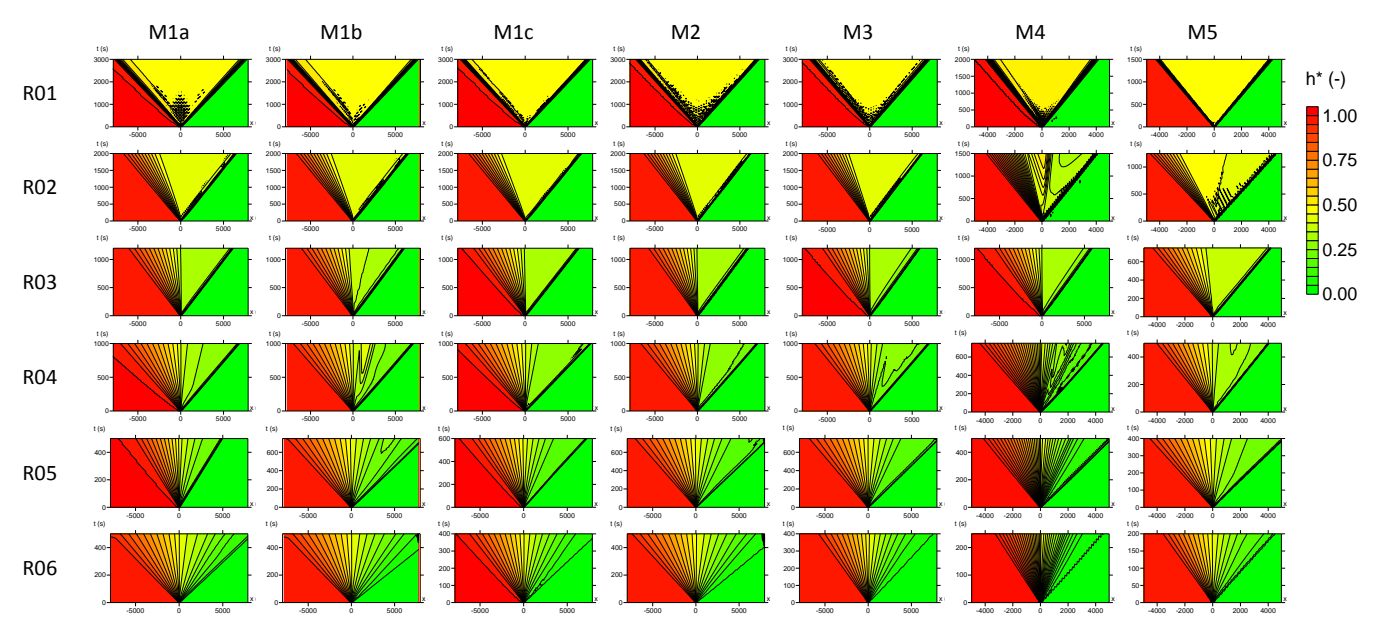

Figure 23: Normalized water depth maps for Models M1-5 and IVPs R01-06. 


\begin{tabular}{|c|c|c|c|c|c|}
\hline IVP & Model & $t(\mathrm{~s})$ & $\int_{-L}^{+L} q \mathrm{~d} x\left(\mathrm{~m}^{3} \mathrm{~s}^{-1}\right)$ & $\frac{\phi_{\Gamma}}{\phi_{\Omega}} \frac{g}{2}\left(h_{L}^{2}-h_{R}^{2}\right) t\left(\mathrm{~m}^{3} \mathrm{~s}^{-1}\right)$ & Ratio \\
\hline \multirow[t]{7}{*}{ R01 } & M1a & 1500 & 88.8 & 88.7 & 1.00 \\
\hline & M1b & 1500 & 88.8 & 88.7 & 1.00 \\
\hline & M1c & 1500 & 88.6 & 88.7 & 1.00 \\
\hline & M2 & 1500 & 82.0 & 82.2 & 1.00 \\
\hline & M3 & 1500 & 77.7 & 77.8 & 1.00 \\
\hline & M4 & 1500 & 56.6 & 56.9 & 1.00 \\
\hline & M5 & 1500 & 114. & $1.14 \times 10^{2}$ & 1.00 \\
\hline \multirow[t]{7}{*}{ R02 } & M1a & 1000 & $7.62 \times 10^{3}$ & $8.83 \times 10^{3}$ & 0.86 \\
\hline & M1b & 1000 & $7.76 \times 10^{3}$ & $8.83 \times 10^{3}$ & 0.88 \\
\hline & M1c & 1000 & $7.92 \times 10^{3}$ & $8.83 \times 10^{3}$ & 0.90 \\
\hline & M2 & 1000 & $7.18 \times 10^{3}$ & $8.18 \times 10^{3}$ & 0.88 \\
\hline & M3 & 1000 & $6.79 \times 10^{3}$ & $7.74 \times 10^{3}$ & 0.88 \\
\hline & M4 & 1000 & $4.21 \times 10^{3}$ & $5.66 \times 10^{3}$ & 0.74 \\
\hline & M5 & 1000 & $1.04 \times 10^{4}$ & $1.13 \times 10^{4}$ & 0.92 \\
\hline \multirow[t]{7}{*}{ R03 } & M1a & 500 & $2.83 \times 10^{4}$ & $3.35 \times 10^{4}$ & 0.80 \\
\hline & M1b & 500 & $2.80 \times 10^{4}$ & $3.53 \times 10^{4}$ & 0.79 \\
\hline & M1c & 500 & $2.95 \times 10^{4}$ & $3.53 \times 10^{4}$ & 0.84 \\
\hline & M2 & 1000 & $5.23 \times 10^{4}$ & $6.54 \times 10^{4}$ & 0.80 \\
\hline & M3 & 1000 & $4.88 \times 10^{4}$ & $6.20 \times 10^{4}$ & 0.79 \\
\hline & M4 & 500 & $1.43 \times 10^{4}$ & $2.26 \times 10^{4}$ & 0.63 \\
\hline & M5 & 500 & $3.89 \times 10^{4}$ & $4.53 \times 10^{4}$ & 0.88 \\
\hline \multirow[t]{7}{*}{ R04 } & M1a & 500 & $1.08 \times 10^{5}$ & $1.46 \times 10^{5}$ & 0.74 \\
\hline & M1b & 500 & $1.08 \times 10^{5}$ & $1.46 \times 10^{5}$ & 0.74 \\
\hline & M1c & 500 & $1.17 \times 10^{5}$ & $1.46 \times 10^{5}$ & 0.80 \\
\hline & M2 & 500 & $1.02 \times 10^{5}$ & $1.35 \times 10^{5}$ & 0.76 \\
\hline & M3 & 500 & $9.54 \times 10^{4}$ & $1.28 \times 10^{5}$ & 0.75 \\
\hline & M4 & 250 & $2.91 \times 10^{4}$ & $9.34 \times 10^{4}$ & 0.62 \\
\hline & M5 & 250 & $1.57 \times 10^{5}$ & $1.87 \times 10^{5}$ & 0.84 \\
\hline \multirow[t]{7}{*}{ R05 } & M1a & 500 & $4.18 \times 10^{5}$ & $5.87 \times 10^{5}$ & 0.71 \\
\hline & M1b & 500 & $4.36 \times 10^{5}$ & $5.87 \times 10^{5}$ & 0.74 \\
\hline & M1c & 500 & $4.59 \times 10^{5}$ & $5.87 \times 10^{5}$ & 0.78 \\
\hline & M2 & 500 & $4.01 \times 10^{5}$ & $5.44 \times 10^{5}$ & 0.74 \\
\hline & M3 & 500 & $3.71 \times 10^{5}$ & $5.15 \times 10^{5}$ & 0.72 \\
\hline & M4 & 250 & $1.13 \times 10^{5}$ & $3.76 \times 10^{5}$ & 0.60 \\
\hline & M5 & 250 & $3.27 \times 10^{5}$ & $7.53 \times 10^{5}$ & 0.87 \\
\hline \multirow[t]{7}{*}{ R06 } & M1a & 500 & $2.43 \times 10^{6}$ & $3.68 \times 10^{6}$ & 0.66 \\
\hline & M1b & 500 & $2.45 \times 10^{6}$ & $3.68 \times 10^{6}$ & 0.67 \\
\hline & M1c & 370 & $2.03 \times 10^{6}$ & $3.68 \times 10^{6}$ & 0.75 \\
\hline & M2 & 400 & $1.90 \times 10^{6}$ & $3.40 \times 10^{6}$ & 0.70 \\
\hline & M3 & 400 & $1.75 \times 10^{6}$ & $3.23 \times 10^{6}$ & 0.68 \\
\hline & M4 & 250 & $6.45 \times 10^{5}$ & $2.36 \times 10^{6}$ & 0.56 \\
\hline & M5 & 250 & $1.91 \times 10^{6}$ & $4.71 \times 10^{6}$ & 0.81 \\
\hline
\end{tabular}

Table 8: Transient momentum dissipation model. Momentum balance results. The dissipation ratio is computed as $1-\frac{\int_{-L}^{+L} q \mathrm{~d} x}{\frac{\phi_{\Gamma}}{\phi_{\Omega}} \frac{g}{2}\left(h_{L}^{2}-h_{R}^{2}\right) t}$.

\section{A.4 Building drag model analysis}

The following figures support and complement the analysis presented in Subsection 5.3. 

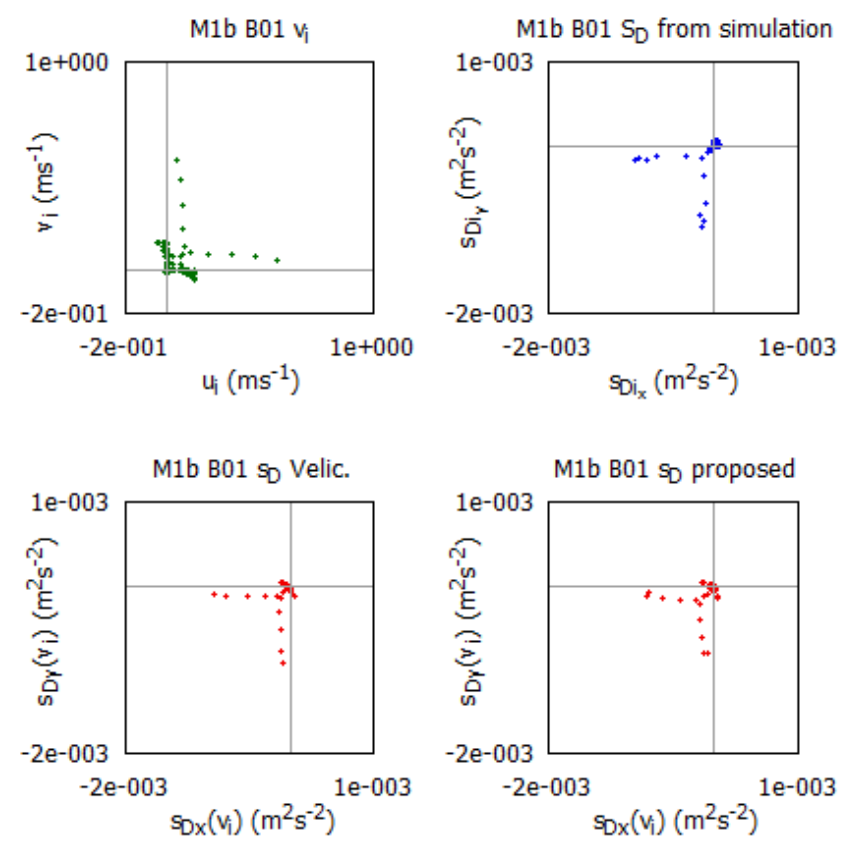

Figure 24: Simulation M1b-B01. Top: domain-averaged velocity vectors $\mathbf{v}_{i}$ (left) and drag vectors $\mathbf{s}_{D, i}$ (right). Bottom: predicted $\mathbf{s}_{D}\left(\mathbf{v}_{i}\right)$ using Velickovic et al.'s model (left) and the proposed formula (right).
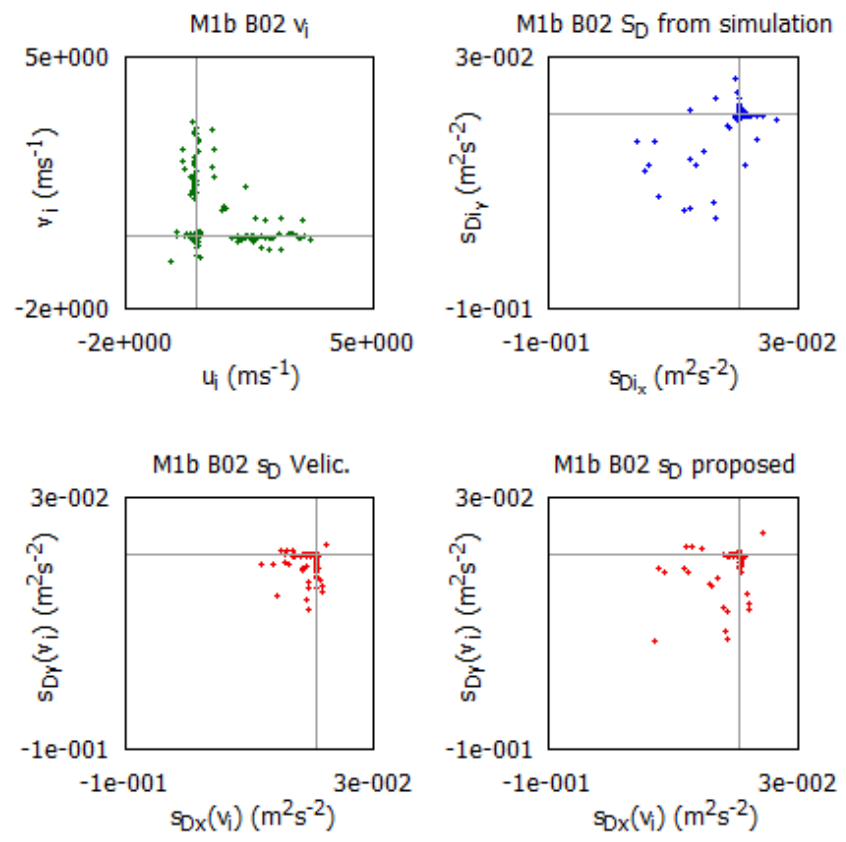

Figure 25: Simulation M1b-B02. Top: domain-averaged velocity vectors $\mathbf{v}_{i}$ (left) and drag vectors $\mathbf{s}_{D, i}$ (right). Bottom: predicted $\mathbf{s}_{D}\left(\mathbf{v}_{i}\right)$ using Velickovic et al.'s model (left) and the proposed formula (right). 

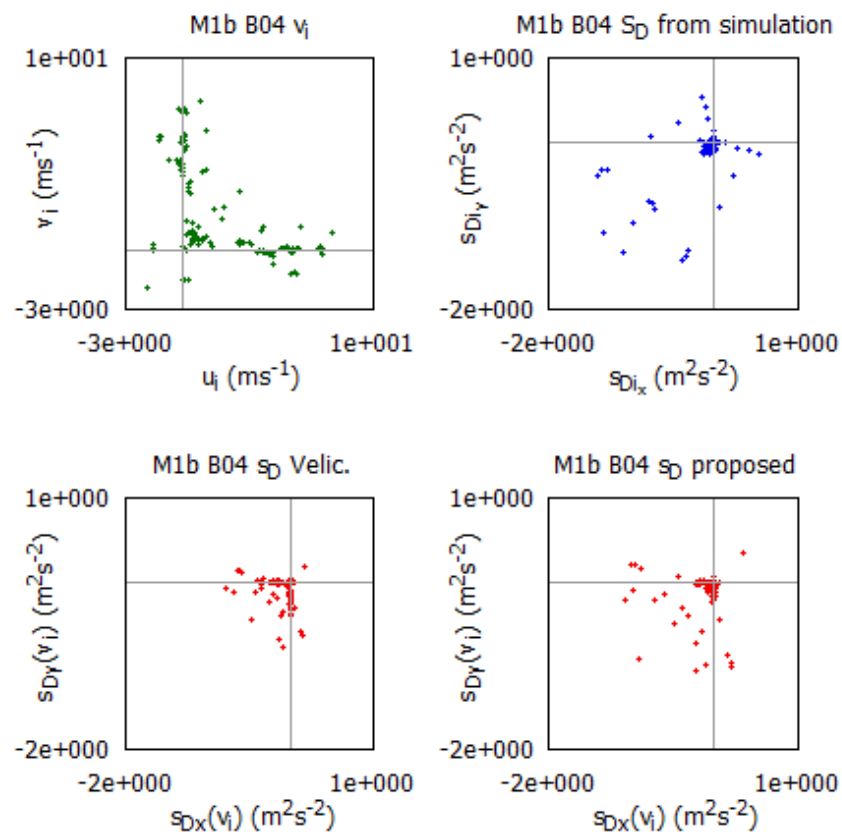

Figure 26: Simulation M1b-B04. Top: domain-averaged velocity vectors $\mathbf{v}_{i}$ (left) and drag vectors $\mathbf{s}_{D, i}$ (right). Bottom: predicted $\mathbf{s}_{D}\left(\mathbf{v}_{i}\right)$ using Velickovic et al.'s model (left) and the proposed formula (right).
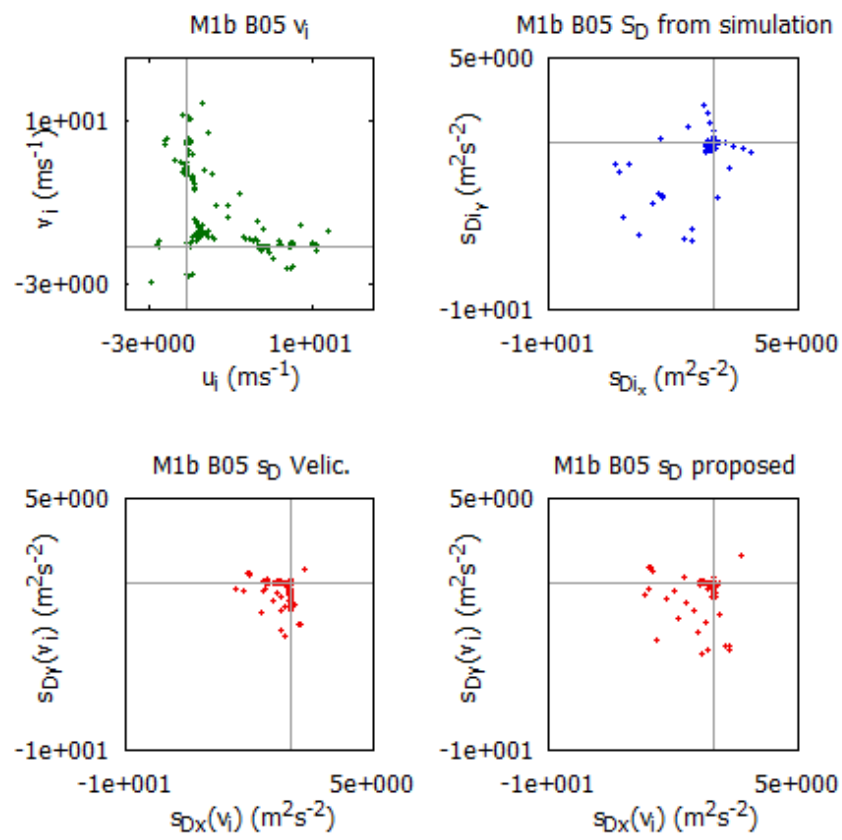

Figure 27: Simulation M1b-B05. Top: domain-averaged velocity vectors $\mathbf{v}_{i}$ (left) and drag vectors $\mathbf{s}_{D, i}$ (right). Bottom: predicted $\mathbf{s}_{D}\left(\mathbf{v}_{i}\right)$ using Velickovic et al.'s model (left) and the proposed formula (right). 

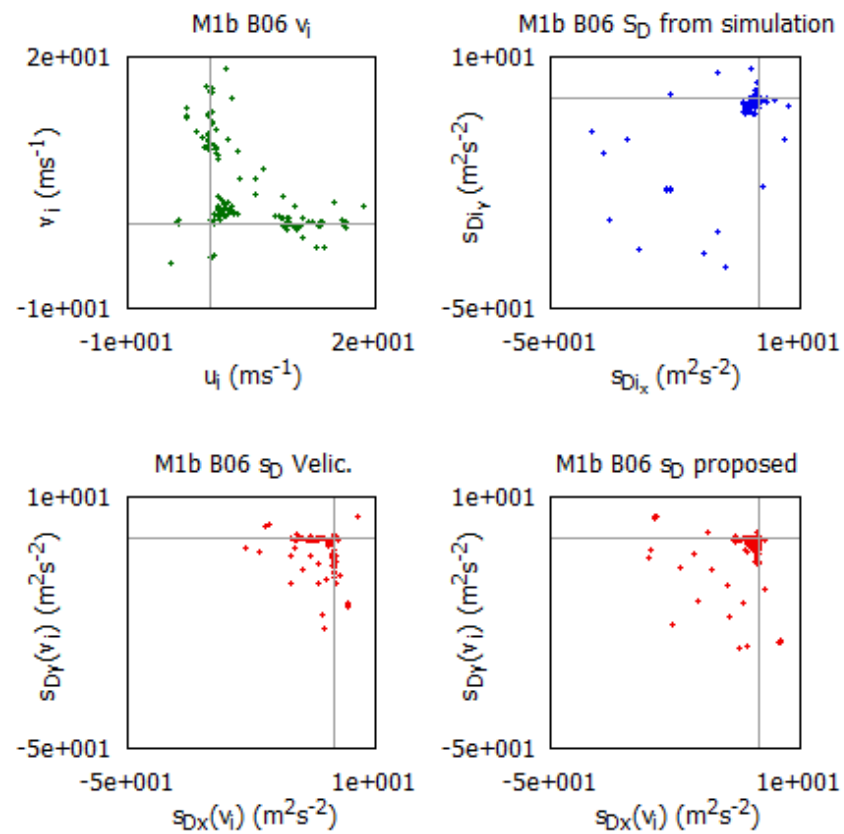

Figure 28: Simulation M1b-B06. Top: domain-averaged velocity vectors $\mathbf{v}_{i}$ (left) and drag vectors $\mathbf{s}_{D, i}$ (right). Bottom: predicted $\mathbf{s}_{D}\left(\mathbf{v}_{i}\right)$ using Velickovic et al.'s model (left) and the proposed formula (right).

\section{References}

[1] Bates, P.D. 2000. Development and testing of a sub-grid scale model for moving boundary hydrodynamic problems in shallow water. Hydrol. Process 14, 2073-2088. DOI: 10.1002/10991085(20000815/30)14:11/12<2073::AID-HYP55>3.0.CO;2-X

[2] Benkhaldoun, F., Elmahi, I., Moumna, A., Seaid, M. 2016. A non-homogeneous Riemann solver for shallow water equations in porous media. Applicable Analysis 95, 2181-2202, DOI: $10.1080 / 00036811.2015 .1067304$

[3] Boris, J.P., Book, D.L. 1973. Flux-corrected transport. I. SHASTA, a fluid transport algorithm that works. Journal of Computational Physics 11, 38-69.

[4] Cea, L., Vazquez-Cendon, M.E. 2010. Unstructured finite volume discretization of two-dimensional depth-averaged shallow water equations with porosity. Int J Numer Methods Fluids 63, 903-30.

[5] Defina, A. 2000. Two-dimensional shallow flow equations for partially dry areas. Water Resour Res 36, 3251-64. DOI: $10.1002 /$ fld.2107

[6] Defina, A., D'Alpaos, L., Mattichio, B. 1994. A new set of equations for very shallow water and partially dry areas suitable to 2D numerical models. In: Molinaro P, Natale L, editors. Proceedings of the specialty conference "Modelling flood propagation over initially dry areas". NY: Am. Soc. Civ. Eng., 82-101.

[7] El Kadi Abderrazak K., Lewicki, L., Paquier, A., Rivière, N., Travin, G. 2011. Divison of critical flow at three-branch open-channel intersection. Journal of Hydraulic Research 49, 231-238. DOI: $10.1080 / 00221686.2011 .558174$ 
[8] Finaud-Guyot, P., Delenne, C., Lhomme, J., Guinot, V., Llovel, C. 2010. An approximate-state Riemann solver for the two-dimensional shallow water equations with porosity. International Journal for Numerical Methods in Fluids 62, 1299-1331. DOI: 10.1002/fld.2066

[9] Ghostine, R., Kesserwani, G., Vazquez, J., Rivière, N., Ghenaim, A., Mose, R. 2009. Simulation of supercritical flow in crossroads: Confrontation of a $2 \mathrm{D}$ and $3 \mathrm{D}$ numerical approaches to experimental results. Computers \& Fluids 38, 425-432. DOI: 10.1016/j.compfluid.2008.05.003

[10] Ghostine, R., Mignot, E., Abdallahn, M., Lawniczac, F., Vazquez, J., Mose, R. Gregoire, C. 2010. Discontinuous Galerkin finite-element method for simulation of flood in crossroads. Journal of Hydraulic Engineering 136, 474-482. DOI: 10.1061/(ASCE)HY.1943-7900.0000209

[11] Guinot, V. 2012. Multiple porosity shallow water models for macroscopic modelling of urban floods. Advances in Water Resources 37, 40-72. DOI: 10.1016/j.advwatres.2011.11.002

[12] Guinot, V. 2017. Consistency and bicharacteristic analysis of integral porosity shallow water models. Explaining model oversensitivity to mesh design. Advances in Water Resources 107, 4355. DOI: $10.1016 /$ j.advwatres.2017.06.008

[13] Guinot, V., Delenne, C. 2014. Macroscopic modelling of urban floods. La Houille Blanche 6, 19-25. DOI: $10.1051 / \mathrm{lhb} / 2014058$

[14] Guinot, V., Sanders, B.F., Schubert, J.E. 2017. Dual integral porosity shallow water model for urban flood modelling, Advances in Water Resources 103, 16-31. DOI: 10.1016/j.advwatres.2017.02.009

[15] Guinot, V., Soares-Frazão, S. 2006. Flux and source term discretisation in two-dimensional shallow water models with porosity on unstructured grids. International Journal for Numerical Methods in Fluids 50, 309-345. DOI: 10.1002/fld.1059

[16] Hervouët, J.M. , Samie, R., Moreau, B., 2000. Modelling urban areas in dam-break floodwave numerical simulations. In: Proceedings of the international seminar and workshop on rescue actions based on dambreak flow analysis, pp. 1-6 . Seinâjoki, Finland.

[17] Kesserwani, G., Vazquez, J., Rivière, N., Liang, Q., Travin, G., Mosé, R. 2010. New approach for predicting flow bifurcation at right-angled open-channel junction. Journal of Hydraulic Engineering 136, 662-668. DOI: 10.1061/(ASCE)HY.1943-7900.0000222

[18] Kim, B., Sanders, B.F., Schubert, J.E., Famiglietti, J.S. 2014. Mesh type tradeoffs in 2D hydrodynamic modeling of flooding with a Godunov-based flow solver. Advances in Water Resources 68, 42-61. DOI: 10.1016/j.advwatres.2014.02.013.

[19] Kim, B., Sanders, B.F., Famiglietti, J.S., Guinot, V. 2015. Urban flood modeling with porous shallow-water equations: a case study of model errors in the presence of anisotropic porosity. J. Hydrol. 523, 680-692 . DOI: 10.1016/j.jhydrol.2015.01.059

[20] Lax, P.D. 1957. Hyperbolic systems of conservation laws II. Communications on pure and Applied Mathematics 10, 537-566.

[21] LeVeque R.J. 2002. Finite volume methods for hyperbolic problems. Cambridge University Press.

[22] Lipeme-Kouyi, G., Rivière, N., Vidalat, V., Becquet, A., Chocat, B., Guinot, V. 2010. Urban flooding: 1D modelling of the distribution of the discharges through cross-road intersections accounting for energy losses. Water Sci. Technol., 61(8), 2021-2028. DOI: 10.2166/wst.2010.133

[23] Mignot, E., Paquier, A., Rivière, N. 2008. Experimental and numerical modeling of symmetrical four-branch supercritical cross junction flow, J. Hydraul. Res. 46(6), 723-738. DOI: 10.1080/00221686.2008.9521918 
[24] Mignot, E., Rivière, N., Perkins, R.J., Paquier A. 2008. Flow patterns in a four branches junction with supercritical flow. J. Hydraul. Eng. 134(6), 701-713. DOI: 10.1061/(ASCE)07339429(2008)134:6(701)

[25] Mignot, E., N. Rivière, N., Paquier, A., Perkins, R.J. 2011. Hydraulic models of the flow distribution in a four branch open channel junction with supercritical flow. J. Hydraul. Eng. 137(3), 289-299. DOI: 10.1061/(ASCE)HY.1943-7900.0000302

[26] Mohamed, K. 2014. A finite volume method for numerical simulation of shallow water models with porosity. Computers \& Fluids 104, 9-19. DOI: 10.1016/j.compfluid.2014.07.020

[27] Momplot, A., Lipeme Kouyi, G., Mignot, E., Rivière, N., Bertrand-Krajewski, J.L. 2017. Typology of the flow structures in dividing open channel flows. Journal of Hydraulic Research 55, 63-71. DOI: $10.1080 / 00221686.2016 .1212409$

[28] Nania, L.S., Gomez M., Dolz J. 2004. Experimental study of the dividing flow in steep street crossings, J. Hydraul. Res., 42(4), 406-412. DOI: 10.1080/00221686.2016.1212409

[29] Özgen, I., Liang, D., Hinkelmann, R. 2016. Shallow water equations with depthdependent anisotropic porosity for subgrid-scale topography. Applied Mathematical Modeling, http://dx.doi.org/10.1016/.j.apm.2015.12.012

[30] Özgen, I., Zhao, J., Liang, D., Hinkelmann, R. 2016. Urban flood modeling using shallow water equations with depth-dependent anisotropic porosity. Journal of Hydrology 541, 1165-1184. DOI: 10.1016/j.jhydrol.2016.08.025

[31] Rivière, N., Perkins R.J., Chocat B., Lecus A. 2006. Flooding flows in city crossroads: 1D modelling and prediction. Water Sci. Technol. 54(6-7), 75-82.

[32] Rivière, N., Travin, G., Perkins, R.J. 2011. Subcritical open channel flows in four branch intersections. Water Resources Research 47, W10517. doi:10.1029/2011WR010504.

[33] Rivière, N., Travin, G., Perkins, R.J. 2014. Transcritical flows in three and four branch openchannel intersections. Journal of Hydraulic Engineering 140: 040114003.

[34] Sanders, B.F., Schubert, J.E., Gallegos, H.A. 2008. Integral formulation of shallow water equations with anisotropic porosity for urban flood modelling. J. Hydrol. 362 19-38. DOI: 10.1016/j.jhydrol.2008.08.009

[35] Soares-Frazão, S., Guinot, V. 2007 An eigenvector-based linear reconstruction scheme for the shallow water equations on two-dimensional unstructured meshes. International Journal for Numerical Methods in Fluids 53, 23-55. DOI: 10.1002/fld.1242

[36] Schubert, J.E., Sanders, B.F. 2012. Building treatments for urban flood inundation models and implications for predictive skill and modeling efficiency. Advances in Water Resources 41, 49-64. DOI: $10.1016 / \mathrm{j}$. advwatres.2012.02.012

[37] Soares-Frazão, S., Lhomme, J., Guinot, V., Zech, Y. 2008. Two-dimensional shallow water models with porosity for urban flood modelling. Journal of Hydraulic Research 46, 45-64. DOI: $10.1080 / 00221686.2008 .9521842$

[38] Velickovic, M., Soares-Frazão, S., Zech, Y. 2017. Steady-flow experiments in urban areas and anisotropic porosity model. Journal of Hydraulic Research 55, 85-100. DOI: $10.1080 / 00221686.2016 .1238013$

[39] Viero, D.P., Valipour, M. 2017. Modeling anisotropy in free-surface overland and shallow inundation flows. Advances in Water Resources 104, 1-14. DOI: 10.1016/j.advwatres.2017.03.007 